Sharif University of Technology
Scientia Iranica
Transactions E: Industrial Engineering
IRAN I I IA

\title{
Bayesian analysis of type-I right censored data using the 3-component mixture of Burr distributions
}

\author{
M. Tahir ${ }^{a, b, *}$, M. Aslam ${ }^{c}$ and Z. Hussain ${ }^{b}$ \\ a. Department of Statistics, Government College University, Faisalabad 38000, Pakistan. \\ b. Department of Statistics, Quaid-i-Azam University, Islamabad 44000, Pakistan. \\ c. Department of Mathematics and Statistics, Riphah International University, Islamabad 44000, Pakistan.
}

Received 4 April 2015; received in revised form 24 June 2015; accepted 26 September 2015

\author{
KEYWORDS \\ Bayesian analysis; \\ Burr distribution; \\ Uniform and Jeffreys' \\ priors; \\ Posterior risk; \\ Predictive interval; \\ Censored data.
}

\begin{abstract}
This study is concerned with the problem of estimating the parameters of a 3-component mixture of Burr distributions using type-I right censored data. The closedform expressions for the Bayes estimators and their posterior risks assuming the noninformative (uniform and Jeffreys') priors under squared-error loss function, precautionary loss function, and DeGroot loss function are derived. Performance of the Bayes estimators for different sample sizes, test termination times (a point of time after which all other tests are terminated), and parametric values under different loss functions is investigated. The posterior predictive distribution for a future observation and the Bayesian predictive interval are constructed. In addition, the limiting expressions for the Bayes estimators and posterior risks are derived. Simulated data sets are designed for the comparisons and the model is finally illustrated using the real data.
\end{abstract}

(C) 2016 Sharif University of Technology. All rights reserved.

\section{Introduction}

Finite mixtures of life distributions have proved to be of considerable interest in terms of their both methodological development and practical applications. Mixture models play a dynamic role in many real-life applications. Saleem [1] discussed that using finite mixture model became necessary when data from individual component densities or conditional distributions were not available, but were available from an overall mixture distribution. The direct applications of mixture models can be seen mostly in industrial engineering, medicine, botany, zoology, paleoanthropology, agriculture, economics, life testing, reliability and survival analysis, etc. A detailed account on type-I and type-II mixture models and their different features is given by $\mathrm{Li}$ [2] and Li and Sedransk [3]. As noted by Tahir and Aslam [4], the mixture of probability density functions

\footnotetext{
*. Corresponding author. Tel.: +92 3015237403

E-mail address: tahirqaustat@yahoo.com (M. Tahir)
}

from the same (different) family (families) is known as type-I (type-II) mixture model. In many applications, the available data can be considered as data from a mixture of two or more distributions. This idea enables us to mix statistical distributions to get a new distribution.

Several authors have extensively applied mixture modeling in different practical problems using classical and Bayesian analyses. For a detailed review of classical estimation techniques, discussion, and applications of mixture modeling, one can refer to [5-40].

Due to time and cost problems, it is sometimes impossible to continue the testing up to the last observation. The values which are greater than the fixed test termination time are taken as censored observations. Due to this limitation, studying censored data is inevitable in lifetime applications. For a detailed review of censoring, one may refer to Romeu [41], Gijbels [42], and Kalbfleisch and Prentice [43] and the references cited therein.

Being able to express a wide range of distribution 
shapes, Burr distribution is considered as a very flexible distribution. It can be fitted to empirical data of different nature. Different sets of its skewness and kurtosis can be covered by different parametric values. Household income, crop prices, insurance risk, travel time, flood levels, and failure data constitute a set of data modeled by the Burr distribution. In his work, Saleem [1] mentioned different cumulative distribution functions, suggested by Burr [44], with a broader range of values of skewness and kurtosis to model any observed data set from a unimodal distribution. Besides this, he discussed twelve forms for cumulative distribution function of the Burr distribution given in Johnson et al. [45]. Saleem [1] also noted that Burr [46,47], Burr and Cislak [48], and Rodriguez [49] gave special attention to one of these forms. A useful discussion on Burr and related distribution is presented by Tadikamalla [50]. Using graphical test, Economou and Caroni [51] explained the utility of Burr distribution. Further enhancing the work on Burr distribution, Saleem [1] presented a 2-component mixture of one-parameter Burr type-XII distribution assuming different priors under squared error loss function.

Motivated by wide application of mixture modeling, in this article, we plan to develop a mixture of Burr distributions for efficient modeling of a given lifetime data. A random variable $Y$ is said to follow a finite mixture distribution with $h$ components if the density function of $Y$ can be written in the form: $f(y)=$ $\sum_{m=1}^{h} p_{m} f_{m}(y)$, where $p_{m}(m=1,2, \ldots, h)$ is the $m$ th mixing proportion such that $p_{h}=1-\sum_{m=1}^{h-1} p_{m}$, i.e. $\sum_{m=1}^{h} p_{m}=1$, and $f_{m}(y)$ is the $m$ th component density function. Under this definition, a finite 3component mixture of Burr distributions with mixing proportions $p_{1}$ and $p_{2}$ has the probability density function (pdf), cumulative distribution function (cdf), and survival function as:

$$
\begin{aligned}
& f(y ; \boldsymbol{\Psi})=p_{1} f_{1}\left(y ; \Psi_{1}\right)+p_{2} f_{2}\left(y ; \Psi_{2}\right) \\
& +\left(1-p_{1}-p_{2}\right) f_{3}\left(y ; \Psi_{3}\right), \\
& p_{1}, p_{2} \geq 0, p_{1}+p_{2} \leq 1, \\
& F(y ; \Psi)=p_{1} F_{1}\left(y ; \Psi_{1}\right)+p_{2} F_{2}\left(y ; \Psi_{2}\right) \\
& +\left(1-p_{1}-p_{2}\right) F_{3}\left(y ; \Psi_{3}\right), \\
& S(y ; \boldsymbol{\Psi})=p_{1} S_{1}\left(y ; \Psi_{1}\right)+p_{2} S_{2}\left(y, \Psi_{2}\right) \\
& +\left(1-p_{1}-p_{2}\right) S_{3}\left(y, \Psi_{3}\right),
\end{aligned}
$$

where, $\boldsymbol{\Psi}=\left(\lambda_{1}, \lambda_{2}, \lambda_{3}, p_{1}, p_{2}\right), \Psi_{m}=\lambda_{m}, m=1,2,3$, and:

$$
f_{m}\left(y ; \Psi_{m}\right)=\lambda_{y}(1+y)^{-\left(\lambda_{m}+1\right)},
$$

$$
0<y<\infty, \lambda_{m}>0, m=1,2,3 .
$$

The cdf, $F_{m}\left(y ; \Psi_{m}\right)$, of the $m$ th component density is given by:

$$
\begin{aligned}
& F_{m}\left(y ; \Psi_{m}\right)=1-(1+y)^{-\lambda_{m}}, \\
& 0<y<\infty, \quad \lambda_{m}>0, m=1,2,3,
\end{aligned}
$$

and $S_{m}\left(y ; \Psi_{m}\right)$, the survival function of the $m$ th component, is written as:

$$
\begin{gathered}
S_{m}\left(y ; \Psi_{m}\right)=1-F_{m}\left(y ; \Psi_{m}\right)=(1+y)^{-\lambda_{m}}, \\
0<y<\infty, \lambda_{m}>0, m=1,2,3 .
\end{gathered}
$$

The rest of the article is organized as follows. The sampling scheme for a 3-component mixture of Burr distributions is defined in Section 2. The expressions for likelihood function and posterior distributions using the non-informative priors are derived in Sections 3 and 4, respectively. In Section 5, the Bayes estimators and posterior risks using the uniform and the Jeffreys' priors under squared error loss function, precautionary loss function, and DeGroot loss function are derived. The posterior predictive distribution and the Bayesian predictive intervals are given in Section 6. The limiting expressions of the Bayes estimators and their posterior risks are derived in Section 7 . The simulation study and the real-life data application are presented in Sections 8 and 9 , respectively. Finally, the conclusion of this study is given in Section 10.

\section{Sampling scheme for a 3-component mixture of Burr distributions}

As defined in Tahir and Aslam [4,52], suppose $n$ units from the 3-component mixture of the Burr distributions, defined in Section 1, are used in a life testing experiment with fixed test termination time $t$. The experiment is performed and it is observed that $r$ out of $n$ units fail until fixed test termination time $t$ is over. The remaining $n-r$ units are still functioning. As defined by Mendenhall and Hader [5], there are many practical situations where only failed objects can be easily recognized as subsets of either subpopulation I or subpopulation II or subpopulation III. For example, based on cause of failure, an engineer may divide a certain failed object as a member of either subpopulation I or subpopulation II or subpopulation III. It may be pointed out that out of $r$ failures, $r_{1}, r_{2}$, and $r_{3}$ failures belong to subpopulation I, subpopulation II, and subpopulation III, respectively, depending upon the reason of failure. Thus, the number of uncensored observations is $r=r_{1}+r_{2}+r_{3}$, and the remaining $n-r$ observations are censored observations. Define $y_{l k}, 0<y_{l k} \leq t$, to be the failure time of the $k$ th unit belonging to the $l$ th subpopulation, where $l=1,2,3$ and $k=1,2, \ldots, r_{l}$. 


\section{The likelihood function}

For a 3-component mixture of the Burr distributions, the likelihood function for the data collected through sampling procedure explained in section 2 can be written as:

$$
\begin{aligned}
& L(\boldsymbol{\Psi} \mid \mathbf{y}) \infty\left\{\boldsymbol{\Pi}_{k=1}^{r_{1}} p_{1} f_{1}\left(y_{1 k}\right)\right\}\left\{\boldsymbol{\Pi}_{k=1}^{r_{2}} p_{2} f_{2}\left(y_{2 k}\right)\right\} \\
&\left\{\boldsymbol{\Pi}_{k=1}^{r_{3}}\left(1-p_{1}-p_{2}\right) f_{3}\left(y_{3 k}\right)\right\}\{S(t)\}^{n-r} .
\end{aligned}
$$

On simplification, the likelihood function of the 3 component mixture of the Burr distribution becomes:

$$
\begin{aligned}
& L(\mathbf{\Psi} \mid \mathbf{y}) \infty\left[\sum_{i=0}^{n-r} \sum_{j=0}^{i}\left(\begin{array}{c}
n-r \\
i
\end{array}\right)\left(\begin{array}{l}
i \\
j
\end{array}\right)\right. \\
& \quad \exp \left\{-\lambda_{1}((n-r-i) \ln (1+t)\right. \\
& \left.\left.+\sum_{k=1}^{r_{1}} \ln \left(1+y_{1 k}\right)\right)\right\} \\
& \quad \exp \left\{-\lambda_{2}\left((i-j) \ln (1+t)+\sum_{k=1}^{r_{2}} \ln \left(1+y_{2 k}\right)\right)\right\} \\
& \quad \exp \left\{-\lambda_{3}\left((j) \ln (1+t)+\sum_{k=1}^{r_{3}} \ln \left(1+y_{3 k}\right)\right)\right\} \\
& \left.\quad \lambda_{1}^{r_{1}} \lambda_{2}^{r_{2}} \lambda_{3}^{r_{3}} p_{1}^{n-r-i+r_{1}} p_{2}^{i-j+r_{2}}\left(1-p_{1}-p_{2}\right)^{j+r_{3}}\right],(8)
\end{aligned}
$$

where, $\mathbf{y}=\left(y_{11}, y_{12}, \ldots, y_{1 r_{1}}, y_{21}, y_{22}, \ldots, y_{2 r_{2}}, y_{31}, y_{32}, \ldots\right.$, $\left.y_{3 r_{3}}\right)$ is the set of observed failure times for the uncensored observations and $\boldsymbol{\Psi}=\left(\lambda_{1}, \lambda_{2}, \lambda_{3}, p_{1}, p_{2}\right)$.

\section{Posterior distributions assuming the non-informative priors}

When no or little prior information is available, the Uniform Prior (UP) and the Jeffreys' Prior (JP) are the most commonly used priors for Bayesian estimation. In this section, the posterior distributions of parameters given in data $\mathbf{y}$ are derived assuming the UP and the JP.

\subsection{Posterior distribution assuming the uniform prior}

Bayes [53] and Geisser [54] proposed that one might consider the uniform distribution for the unknown parameters of interest (see Tahir et al. [4] and Tahir and Aslam [52]). We assume the improper UP for the unknown component parameter $\lambda_{m}$, i.e. $\lambda_{m} \sim$ Uniform $(0, \infty), m=1,2,3$, and the UP over the interval $(0,1)$ for the unknown proportion parameter $p_{s}$, i.e. $p_{s} \sim$ Uniform $(0,1), s=1,2$. Assuming the independence of parameters, the joint prior distribution of parameters $\lambda_{1}, \lambda_{2}, \lambda_{3}, p_{1}$, and $p_{2}$ is denoted by $\pi_{1}(\Psi) \propto 1$ (see $[4,52]$ ). Thus, the joint posterior distribution of parameters $\lambda_{1}, \lambda_{2}, \lambda_{3}, p_{1}$, and $p_{2}$ for the given data $\mathbf{y}$, assuming the UP, is defined by Eqs. (9) and (10) as shown in Box I, where:

$$
\begin{aligned}
& A_{11}=r_{1}+1, \quad A_{21}=r_{2}+1 \quad A_{31}=r_{3}+1, \sum_{11}=(n-r-i) \ln (1+t)+\sum_{k=1}^{r_{1}} \ln \left(1+y_{1 k}\right), \\
& B_{21}=(i-j) \ln (1+t)+\sum_{k=1}^{r_{2}} \ln \left(1+y_{2 k}\right), \\
& B_{31}=(j) \ln (1+t)+\sum_{k=1}^{r_{3}} \ln \left(1+y_{3 k}\right), \\
& A_{01}=n-r-i+r_{1}+1, \quad B_{01}=i-j+r_{2}+1, \\
& C_{01}=j+r_{3}+1, \\
& \Omega_{1}=\Gamma\left(A_{11}\right) \Gamma\left(A_{21}\right) \Gamma\left(A_{31}\right) \sum_{i=0}^{n-r} \sum_{j=0}^{i}\left(\begin{array}{c}
n-r \\
i
\end{array}\right)\left(\begin{array}{l}
i \\
j
\end{array}\right) \\
& B\left(A_{01}, B_{01}, C_{01}\right) B_{11}^{-A_{11}} B_{21}^{-A_{21}} B_{31}^{-A_{31}} .
\end{aligned}
$$

The marginal posterior distributions of parameters $\lambda_{1}, \lambda_{2}, \lambda_{2}, p_{1}$, and $p_{2}$ using the UP are derived as:

$$
\begin{aligned}
q_{1}(\boldsymbol{\Psi} \mid \mathbf{y}) & =\frac{L(\mathbf{\Psi} \mid \mathbf{y}) \pi_{1}(\mathbf{\Psi})}{\int_{\Psi} L(\mathbf{\Psi} \mid \mathbf{y}) \pi_{1}(\mathbf{\Psi}) d \mathbf{\Psi}} \\
q_{1}(\boldsymbol{\Psi} \mid \mathbf{y}) & =\frac{\sum_{i=0}^{n-r} \sum_{j=0}^{i}\left(\begin{array}{c}
n-r \\
i
\end{array}\right)\left(\begin{array}{c}
i \\
j
\end{array}\right) \exp \left(-B_{11} \lambda_{1}\right) \exp \left(-B_{21} \lambda_{2}\right) \exp \left(-B_{31} \lambda_{3}\right) p_{1}^{A_{01}-1} p_{2}^{B_{01}-1}\left(1-p_{1}-p_{2}\right)^{C_{01}-1}}{\Omega_{1} \lambda_{1}^{1-A_{11}} \lambda_{2}^{1-A_{21}} \lambda_{3}^{1-A_{31}}}
\end{aligned}
$$




$$
\begin{aligned}
g_{1}\left(\lambda_{1} \mid \mathbf{y}\right)= & \frac{\Gamma\left(A_{21}\right) \Gamma\left(A_{31}\right)}{\Omega_{1}} \sum_{i=0}^{n-r} \sum_{j=0}^{i}\left(\begin{array}{c}
n-r \\
i
\end{array}\right)\left(\begin{array}{c}
i \\
j
\end{array}\right) \\
& B_{21}^{-A_{21}} B_{31}^{-A_{31}} B\left(A_{01}, C_{01}\right) B\left(B_{01}, A_{01}\right. \\
& \left.+C_{01}\right) \lambda_{1}^{A_{11}-1} \exp \left(-B_{11} \lambda_{1}\right), \\
& \lambda_{1}>0
\end{aligned}
$$$$
g_{1}\left(\lambda_{2} \mid \mathbf{y}\right)=\frac{\Gamma\left(A_{11}\right) \Gamma\left(A_{31}\right)}{\Omega_{1}} \sum_{i=0}^{n-r} \sum_{j=0}^{i}\left(\begin{array}{c}
n-r \\
i
\end{array}\right)\left(\begin{array}{l}
i \\
j
\end{array}\right)
$$$$
B_{11}^{-A_{11}} B_{31}^{-A_{31}} B\left(A_{01}, C_{01}\right) B\left(B_{01}, A_{01}\right.
$$$$
\left.+C_{01}\right) \lambda_{2}^{A_{21}-1} \exp \left(-B_{21} \lambda_{2}\right)
$$$$
\lambda_{2}>0,
$$$$
g_{1}\left(\lambda_{3} \mid \mathbf{y}\right)=\frac{\Gamma\left(A_{21}\right) \Gamma\left(A_{31}\right)}{\Omega_{1}} \sum_{i=0}^{n-r} \sum_{j=0}^{i}\left(\begin{array}{c}
n-r \\
i
\end{array}\right)\left(\begin{array}{l}
i \\
j
\end{array}\right)
$$$$
B_{11}^{-A_{11}} B_{21}^{-A_{21}} B\left(A_{01}, C_{01}\right) B\left(B_{01}, A_{01}\right.
$$$$
\left.+C_{01}\right) \lambda_{3}^{A_{31}-1} \exp \left(-B_{31} \lambda_{3}\right),
$$$$
\lambda_{3}>0,
$$

$$
g_{1}\left(p_{1} \mid \mathbf{y}\right)=\frac{\Gamma\left(A_{11}\right) \Gamma\left(A_{21}\right) \Gamma\left(A_{31}\right)}{\Omega_{1}} \sum_{i=0}^{n-r} \sum_{j=0}^{i}\left(\begin{array}{c}
n-r \\
i
\end{array}\right)\left(\begin{array}{l}
i \\
j
\end{array}\right)
$$$$
B_{11}^{-A_{11}} B_{21}^{-A_{21}} B_{31}^{-A_{31}} B\left(B_{01}, C_{01}\right) p_{1}^{A_{01}-1}
$$$$
\left(1-p_{1}\right)^{B_{01}+C_{01}+1} \text {, }
$$$$
0<p_{1}<1,
$$

$$
\begin{aligned}
g_{1}\left(p_{2} \mid \mathbf{y}\right)= & \frac{\Gamma\left(A_{11}\right) \Gamma\left(A_{21}\right) \Gamma\left(A_{31}\right)}{\Omega_{1}} \sum_{i=0}^{n-r} \sum_{j=0}^{i}\left(\begin{array}{c}
n-r \\
i
\end{array}\right)\left(\begin{array}{l}
i \\
j
\end{array}\right) \\
& B_{11}^{-A_{11}} B_{21}^{-A_{21}} B_{31}^{-A_{31}} B\left(A_{01}, C_{01}\right) p_{2}^{B_{01}-1} \\
& \left(1-p_{2}\right)^{A_{01}+C_{01}-1}, \\
& 0<p_{2}<1 .
\end{aligned}
$$
in Box II, where:

$$
\begin{aligned}
A_{12}= & r_{1}, \quad A_{22}=r_{2}, \\
A_{32}= & r_{3}, \\
B_{12}= & (n-r-i) \ln (1+t)+\sum_{k=1}^{r_{1}} \ln \left(1+y_{1 k}\right), \\
B_{22}= & (i-j) \ln (1+t)+\sum_{k=1}^{r_{2}} \ln \left(1+y_{2 k}\right), \\
B_{32}= & (j) \ln (1+t)+\sum_{k=1}^{r_{3}} \ln \left(1+y_{3 k}\right), \\
A_{02}= & n-r-i+r_{1}+1, \\
B_{02}= & i-j+r_{2}+1, \\
C_{02}= & j+r_{3}+1, \\
\Omega_{2}= & \Gamma\left(A_{12}\right) \Gamma\left(A_{22}\right) \Gamma\left(A_{32}\right) \sum_{i=0}^{n-r} \sum_{j=0}^{i}\left(\begin{array}{c}
n-r \\
i
\end{array}\right)\left(\begin{array}{l}
i \\
j
\end{array}\right) \\
& B\left(A_{02}, B_{02}, C_{02}\right) B_{12}^{-A_{12}} B_{22}^{-A_{22}} B_{32}^{-A_{32} .} .
\end{aligned}
$$

The marginal posterior distributions of parameters $\lambda_{1}, \lambda_{2}, \lambda_{3}, p_{1}$, and $p_{2}$ using the JP are worked out as:

$$
\begin{aligned}
g_{2}\left(\lambda_{1} \mid \mathbf{y}\right)= & \frac{\Gamma\left(A_{22}\right) \Gamma\left(A_{32}\right)}{\Omega_{2}} \sum_{i=0}^{n-r} \sum_{j=0}^{i}\left(\begin{array}{c}
n-r \\
i
\end{array}\right)\left(\begin{array}{l}
i \\
j
\end{array}\right) \\
& B_{22}^{-A_{22}} B_{32}^{-A_{32}} B\left(A_{02}, C_{02}\right) B\left(B_{02}, A_{02}\right. \\
& \left.+C_{02}\right) \lambda_{1}^{A_{12}-1} \exp \left(-B_{12} \lambda_{1}\right), \\
& \lambda_{1}>0, \\
g_{2}\left(\lambda_{2} \mid \mathbf{y}\right)= & \frac{\Gamma\left(A_{12}\right) \Gamma\left(A_{32}\right)}{\Omega_{2}} \sum_{i=0}^{n-r} \sum_{j=0}^{i}\left(\begin{array}{c}
n-r \\
i
\end{array}\right)\left(\begin{array}{c}
i \\
j
\end{array}\right) \\
& B_{12}^{-A_{12}} B_{32}^{-A_{32}} B\left(A_{02}, C_{02}\right) B\left(B_{02}, A_{02}\right. \\
& \left.+C_{02}\right) \lambda_{2}^{A_{22}-1} \exp \left(-B_{22} \lambda_{2}\right), \\
& \lambda_{2}>0,
\end{aligned}
$$




$$
\begin{aligned}
q_{2}(\boldsymbol{\Psi} \mid \mathbf{y}) & =\frac{L(\mathbf{\Psi} \mid \mathbf{y}) \pi_{2}(\mathbf{\Psi})}{\int_{\Psi} L(\mathbf{\Psi} \mid \mathbf{y}) \pi_{2}(\mathbf{\Psi}) d \mathbf{\Psi}}, \\
q_{2}(\mathbf{\Psi} \mid \mathbf{y}) & =\frac{\sum_{i=1}^{n-r} \sum_{j=0}^{i}\left(\begin{array}{c}
n-r \\
i
\end{array}\right)\left(\begin{array}{c}
i \\
j
\end{array}\right) \exp \left(-B_{12} \lambda_{1}\right) \exp \left(-B_{22} \lambda_{2}\right) \exp \left(-B_{32} \lambda_{3}\right) p_{1}^{A_{02}-1} p_{2}^{B_{01}-1}\left(1-p_{1}-p_{2}\right)^{C_{02}-1}}{\Omega_{2} \lambda_{1}^{1-A_{12}} \lambda_{2}^{1-A_{22}} \lambda_{3}^{1-A_{32}}}
\end{aligned}
$$

\section{Box II}

Table 1. Bayes estimators and posterior risks under SELF, PLF, and DLF.

\begin{tabular}{ccc}
\hline Loss function & Bayes estimators & Posterior risks \\
\hline $\operatorname{SELF}=L(\lambda, d)=(\lambda-d)^{2}$ & $\hat{d}=E_{\lambda \mid \mathbf{y}}(\lambda)$ & $\rho(\hat{d})=E_{\lambda \mid \mathbf{y}}\left(\lambda^{2}\right)-\left\{E_{\lambda \mid \mathbf{y}(\lambda)}\right\}^{2}$ \\
$\operatorname{PLF}=L(\lambda, d)=\frac{(\lambda-d)^{2}}{d}$ & $\hat{d}=\left\{E_{\lambda \mid \mathbf{y}}\left(\lambda^{2}\right)\right\}^{\frac{1}{2}}$ & $\rho(\hat{d})=2\left\{E_{\lambda \mid \mathbf{y}}\left(\lambda^{2}\right)\right\}^{\frac{1}{2}}-2 E_{\lambda \mid \mathbf{y}}(\lambda)$ \\
$\operatorname{DLF}=L(\lambda, d)=\left(\frac{\lambda-d}{d}\right)^{2}$ & $\hat{d}=\left\{E_{\lambda \mid \mathbf{y}}(\lambda)\right\}^{-1} E_{\lambda \mid \mathbf{y}}\left(\lambda^{2}\right)$ & $\rho(\hat{d})=1-\left\{E_{\lambda \mid \mathbf{y}}(\lambda)\right\}^{2}\left\{E_{\lambda \mid \mathbf{y}}\left(\lambda^{2}\right)\right\}^{-1}$ \\
\hline
\end{tabular}

$$
\begin{gathered}
g_{2}\left(\lambda_{3} \mid \mathbf{y}\right)=\frac{\Gamma\left(A_{22}\right) \Gamma\left(A_{32}\right)}{\Omega_{2}} \sum_{i=0}^{n-r} \sum_{j=0}^{i}\left(\begin{array}{c}
n-r \\
i
\end{array}\right)\left(\begin{array}{l}
i \\
j
\end{array}\right) \\
B_{12}^{-A_{12}} B_{22}^{-A_{22}} B\left(A_{02}, C_{02}\right) B\left(B_{02}, A_{02}\right. \\
\left.+C_{02}\right) \lambda_{3}^{A_{32}-1} \exp \left(-B_{32} \lambda_{3}\right), \\
\lambda_{3}>0
\end{gathered}
$$

$$
\begin{aligned}
g_{2}\left(p_{1} \mid \mathbf{y}\right)= & \frac{\Gamma\left(A_{12}\right) \Gamma\left(A_{22}\right) \Gamma\left(A_{32}\right)}{\Omega_{2}} \sum_{i=0}^{n-r} \sum_{j=0}^{i}\left(\begin{array}{c}
n-r \\
i
\end{array}\right) \\
& \left(\begin{array}{c}
i \\
j
\end{array}\right) B_{12}^{-A_{12}} B_{22}^{-A_{22}} B_{32}^{-A_{32}} B\left(B_{02}, C_{02}\right) \\
& p_{1}^{A_{02}-1}\left(1-p_{1}\right)^{B_{02}+C_{02}-1}, \\
& 0<p_{1}<1,
\end{aligned}
$$

$$
g_{2}\left(p_{2} \mid \mathbf{y}\right)=\frac{\Gamma\left(A_{12}\right) \Gamma\left(A_{22}\right) \Gamma\left(A_{32}\right)}{\Omega_{2}} \sum_{i=0}^{n-r} \sum_{j=0}^{i}\left(\begin{array}{c}
n-r \\
i
\end{array}\right)
$$

$$
\begin{aligned}
& \left(\begin{array}{l}
i \\
j
\end{array}\right) B_{12}^{-A_{12}} B_{22}^{-A_{22}} B_{32}^{-A_{32}} B\left(A_{02}, C_{02}\right) \\
& p_{2}^{B_{02}-1}\left(1-p_{2}\right)^{A_{02}+C_{02}-1}, \\
& 0<p_{2}<1 .
\end{aligned}
$$

\section{Bayesian estimation}

In this section, we present the derivation of the Bayes estimators and posterior risks using the UP and the JP under three different loss functions, namely, Squared Error Loss Function (SELF), Precautionary Loss Function (PLF), and DeGroot Loss Function (DLF). The SELF, defined as:

$$
L=(\lambda, d)=(\lambda-d)^{2},
$$

was introduced by Legendre [57] to develop the least square theory. Norstrom [58] discussed an asymmetric PLF and also introduced a special case of a general class of PLFs, which was defined as $L(\lambda, d)=\frac{(\lambda-d)^{2}}{d}$. The DLF was presented by DeGroot [59] and was defined as $L(\lambda, d)=\left(\frac{\lambda-d}{d}\right)^{2}$ (see [4,52]). For a given prior, the Bayes estimators and posterior risks under SELF, PLF, and DLF are given in Table 1.

\subsection{Expressions for the Bayes estimators and posterior risks assuming the UP and the JP under SELF}

The expressions for the Bayes estimators and posterior risks are derived as follows:

$$
\begin{aligned}
\hat{\lambda}_{1 v}= & \frac{\Gamma\left(A_{1 v}+1\right) \Gamma\left(A_{2 v}\right) \Gamma\left(A_{3 v}\right)}{\Omega_{v}} \sum_{i=0}^{n-r} \sum_{j=0}^{i}\left(\begin{array}{c}
n-r \\
i
\end{array}\right)\left(\begin{array}{l}
i \\
j
\end{array}\right) \\
& B_{1 v}^{-\left(A_{1 v}+1\right)} B_{2 v}^{-A_{2 v}} B_{3 v}^{-A_{3 v}} B\left(A_{0 v}, C_{0 v}\right) \\
& B\left(B_{0 v}, A_{0 v}+C_{0 v}\right), \\
\hat{\lambda}_{2 v}= & \frac{\Gamma\left(A_{1 v}\right) \Gamma\left(A_{2 v}+1\right) \Gamma\left(A_{3 v}\right)}{\Omega_{v}} \sum_{i=0}^{n-r} \sum_{j=0}^{i}\left(\begin{array}{c}
n-r \\
i
\end{array}\right)\left(\begin{array}{c}
i \\
j
\end{array}\right) \\
& B_{1 v}^{-A_{1 v}} B_{2 v}^{-\left(A_{2 v}+1\right)} B_{3 v}^{-A_{3 v}} B\left(A_{0 v}, C_{0 v}\right) \\
& B\left(B_{0 v}, A_{0 v}+C_{0 v}\right),
\end{aligned}
$$




$$
\begin{aligned}
\hat{\lambda}_{3 v}= & \frac{\Gamma\left(A_{1 v}\right) \Gamma\left(A_{2 v}\right) \Gamma\left(A_{3 v}+1\right)}{\Omega_{v}} \sum_{i=0}^{n-r} \sum_{j=0}^{i}\left(\begin{array}{c}
n-r \\
i
\end{array}\right)(i j) \\
& B_{1 v}^{-A_{1 v}} B_{2 v}^{-A_{2 v}} B_{3 v}^{-\left(A_{3 v}+1\right)} B\left(A_{0 v}, C_{0 v}\right) \\
& B\left(B_{0 v}, A_{0 v}+C_{0 v}\right),
\end{aligned}
$$$$
\hat{p}_{1 v}=\frac{\Gamma\left(A_{1 v}\right) \Gamma\left(A_{2 v}\right) \Gamma\left(A_{3 v}\right)}{\Omega_{v}} \sum_{i=0}^{n-r} \sum_{j=0}^{i}\left(\begin{array}{c}
n-r \\
i
\end{array}\right)\left(\begin{array}{l}
i \\
j
\end{array}\right)
$$$$
B_{1 v}^{-A_{1 v}} B_{2 v}^{-A_{2 v}} B_{3 v}^{-A_{3 v}} B\left(B_{0 v}, C_{0 v}\right)
$$$$
B\left(A_{0 v}+1, B_{0 v}+C_{0 v}\right),
$$$$
\hat{p}_{2 v}=\frac{\Gamma\left(A_{1 v}\right) \Gamma\left(A_{2 v}\right) \Gamma\left(A_{3 v}\right)}{\Omega_{v}} \sum_{i=0}^{n-r} \sum_{j=0}^{i}\left(\begin{array}{c}
n-r \\
i
\end{array}\right)\left(\begin{array}{l}
i \\
j
\end{array}\right)
$$$$
B_{1 v}^{-A_{1 v}} B_{2 v}^{-A_{2 v}} B_{3 v}^{-A_{3 v}} B\left(A_{0 v}, C_{0 v}\right)
$$$$
B\left(B_{0 v}+1, A_{0 v}+C_{0 v}\right),
$$$$
\rho\left(\hat{\lambda}_{1 v}\right)=\frac{\Gamma\left(A_{1 v}+2\right) \Gamma\left(A_{2 v}\right) \Gamma\left(A_{3 v}\right)}{\Omega_{v}}
$$$$
\sum_{i=0}^{n-r} \sum_{j=0}^{i}\left(\begin{array}{c}
n-r \\
i
\end{array}\right)\left(\begin{array}{l}
i \\
j
\end{array}\right)
$$$$
B_{1 v}^{-\left(A_{1 v}+2\right)} B_{2 v}^{-A_{2 v}} B_{3 v}^{-A_{3 v}} B\left(A_{0 v}, C_{0 v}\right)
$$$$
B\left(B_{0 v}, A_{0 v}+C_{0 v}\right)
$$$$
-\left\{\frac{\Gamma\left(A_{1 v}+1\right) \Gamma\left(A_{2 v}\right) \Gamma\left(A_{3 v}\right)}{\Omega_{v}} \sum_{i=0}^{n-r} \sum_{j=0}^{i}\right.
$$$$
\left(\begin{array}{c}
n-r \\
i
\end{array}\right)\left(\begin{array}{c}
i \\
j
\end{array}\right) B_{1 v}^{-\left(A_{1 v}+1\right)} B_{2 v}^{-A_{2 v}} B_{3 v}^{-A_{3 v}}
$$$$
\left.B\left(A_{0 v}, C_{0 v}\right) B\left(B_{0 v}, A_{0 v}+C_{0 v}\right)\right\}^{2},
$$$$
\rho\left(\hat{\lambda}_{2 v}\right)=\frac{\Gamma\left(A_{1 v}\right) \Gamma\left(A_{2 v}+2\right) \Gamma\left(A_{3 v}\right)}{\Omega_{v}} \sum_{i=0}^{n-r} \sum_{j=0}^{i}
$$$$
\left(\begin{array}{c}
n-r \\
i
\end{array}\right)\left(\begin{array}{c}
i \\
j
\end{array}\right) B_{1 v}^{-A_{1 v}} B_{2 v}^{-\left(A_{2 v}+2\right)} B_{3 v}^{-A_{3 v}}
$$$$
B\left(A_{0 v}, C_{0 v}\right) B\left(B_{0 v}, A_{0 v}+C_{0 v}\right)
$$

$$
\begin{aligned}
& -\left\{\frac{\Gamma\left(A_{1 v}\right) \Gamma\left(A_{2 v}+1\right) \Gamma\left(A_{3 v}\right)}{\Omega_{v}} \sum_{i=0}^{n-r} \sum_{j=0}^{i}\right. \\
& \left(\begin{array}{c}
n-r \\
i
\end{array}\right)\left(\begin{array}{c}
i \\
j
\end{array}\right) B_{1 v}^{-A_{1 v}} B_{2 v}^{-\left(A_{2 v}+1\right)} B_{3 v}^{-A_{3 v}} \\
& \left.B\left(A_{0 v}, C_{0 v}\right) B\left(B_{0 v}, A_{0 v}+C_{0 v}\right)\right\}^{2}
\end{aligned}
$$$$
\rho\left(\hat{\lambda}_{3 v}\right)=\frac{\Gamma\left(A_{1 v}\right) \Gamma\left(A_{2 v}\right) \Gamma\left(A_{3 v}+2\right)}{\Omega_{v}} \sum_{i=0}^{n-r} \sum_{j=0}^{i}
$$$$
\left(\begin{array}{c}
n-r \\
i
\end{array}\right)\left(\begin{array}{l}
i \\
j
\end{array}\right) B_{1 v}^{-A_{1 v}} B_{2 v}^{-A_{2 v}} B_{3 v}^{-\left(A_{3 v}+2\right)}
$$$$
B\left(A_{0 v}, C_{0 v}\right) B\left(B_{0 v}, A_{0 v}+C_{0 v}\right)
$$$$
-\left\{\frac{\Gamma\left(A_{1 v}\right) \Gamma\left(A_{2 v}\right) \Gamma\left(A_{3 v}+1\right)}{\Omega_{v}} \sum_{i=0}^{n-r} \sum_{j=0}^{i}\right.
$$$$
\left(\begin{array}{c}
n-r \\
i
\end{array}\right)\left(\begin{array}{c}
i \\
j
\end{array}\right) B_{1 v}^{-A_{1 v}} B_{2 v}^{-A_{2 v}} B_{3 v}^{-\left(A_{3 v}+1\right)}
$$

$$
\begin{aligned}
& \left.B\left(A_{0 v}, C_{0 v}\right) B\left(B_{0 v}, A_{0 v}+C_{0 v}\right)\right\}^{2}, \\
\left.\hat{p}_{1 v}\right)= & \frac{\Gamma\left(A_{1 v}\right) \Gamma\left(A_{2 v}\right) \Gamma\left(A_{3 v}\right)}{\Omega_{v}} \sum_{i=0}^{n-r} \sum_{j=0}^{i}\left(\begin{array}{c}
n-r \\
i
\end{array}\right)\left(\begin{array}{c}
i \\
j
\end{array}\right) \\
& B_{1 v}^{-A_{1 v}} B_{2 v}^{-A_{2 v}} B_{3 v}^{-A_{3 v}} B\left(B_{0 v}, C_{0 v}\right) \\
& B\left(A_{0 v}+2, B_{0 v}+C_{0 v}\right) \\
& -\left\{\frac{\Gamma\left(A_{1 v}\right) \Gamma\left(A_{2 v}\right) \Gamma\left(A_{3 v}\right)}{\Omega_{v}} \sum_{i=0}^{n-r} \sum_{j=0}^{i}\right. \\
& \left(\begin{array}{c}
n-r \\
i
\end{array}\right)\left(\begin{array}{c}
i \\
j
\end{array}\right) B_{1 v}^{-A_{1 v}} B_{2 v}^{-A_{2 v}} B_{3 v}^{-A_{3 v}} B\left(B_{0 v}, C_{0 v}\right) \\
& \left.B\left(A_{0 v}+1, B_{0 v}+C_{0 v}\right)\right\}^{2},
\end{aligned}
$$

$$
\begin{aligned}
\rho\left(\hat{p}_{2 v}\right)= & \frac{\Gamma\left(A_{1 v}\right) \Gamma\left(A_{2 v}\right) \Gamma\left(A_{3 v}\right)}{\Omega_{v}} \\
& \sum_{i=0}^{n-r} \sum_{j=0}^{i}\left(\begin{array}{c}
n-r \\
i
\end{array}\right)\left(\begin{array}{c}
i \\
j
\end{array}\right) \\
& B_{1 v}^{-A_{1 v}} B_{2 v}^{-A_{2 v}} B_{3 v}^{-A_{3 v}} B\left(A_{0 v}, C_{0 v}\right) \\
& B\left(B_{0 v}+2, A_{0 v}+C_{0 v}\right)
\end{aligned}
$$




$$
\begin{aligned}
& -\left\{\frac{\Gamma\left(A_{1 v}\right) \Gamma\left(A_{2 v}\right) \Gamma\left(A_{3 v}\right)}{\Omega_{v}} \sum_{i=0}^{n-r} \sum_{j=0}^{i}\right. \\
& \left(\begin{array}{c}
n-r \\
i
\end{array}\right)\left(\begin{array}{c}
i \\
j
\end{array}\right) B_{1 v}^{-A_{1 v}} B_{2 v}^{-A_{2 v}} B_{3 v}^{-A_{3 v}} B\left(A_{0 v}, C_{0 v}\right) \\
& \left.B\left(B_{0 v}+1, A_{0 v}+C_{0 v}\right)\right\}^{2},
\end{aligned}
$$

where $v=1$ for the UP and $v=2$ for the JP.

Similarly, the Bayes estimators and posterior risks using the UP and the JP under PLF and DLF can also be derived. For the sake of brevity, we have not presented these expressions; but, these expressions are available in the studies by the first author.

\section{Posterior predictive distribution and Bayesian predictive interval}

The posterior predictive distribution contains the information about the future observation, $X=Y_{n+1}$, of a random variable given in the data, $\mathbf{y}$, already observed. Dependent on the observed data, it is defined as the distribution of a new independent and identical future observation drawn from the same population. It is normally used in a Bayesian framework. Using the entire posterior distribution of the parameter(s), a probability distribution over an interval is derived as a posterior predictive distribution of the future observation conditional on the observed data. To be more specific, by marginalizing the posterior distribution over the parameter(s), posterior predictive distribution of future observation can be derived. Arnold and Press [60], Al-Hussaini et al. [61], Al-Hussaini and Ahmad [62], Bolstad [63], and Bansal [64] have given a detailed discussion on prediction and predictive distribution under the Bayesian paradigm. We, now, present the derivation of posterior predictive distribution and Bayesian predictive interval.

The posterior predictive distribution of a future observation, $X=Y_{n+1}$, for the given data, y, assuming the UP and the JP, is written as:

$$
\begin{gathered}
f(x \mid \underline{\mathrm{y}})=\int_{p_{2}} \int_{p_{1}} \int_{\lambda_{3}} \int_{\lambda_{2}} \int_{\lambda_{1}} f(x \mid \mathbf{\Psi}) q_{v}(\mathbf{\Psi} \mid \mathbf{y}) \\
d \lambda_{1} d \lambda_{2} d \lambda_{3} d p_{1} d p_{2},
\end{gathered}
$$

where:

$$
\begin{aligned}
& f(x \mid \Psi)=p_{1} f_{1}\left(x ; \Psi_{1}\right)+p_{2} f_{2}\left(x ; \Psi_{2}\right)+ \\
& \left(1-p_{1}-p_{2}\right) f_{3}\left(x ; \Psi_{3}\right), \\
& f_{m}\left(x ; \Psi_{m}\right)=\lambda_{m}(1+x)^{-\left(\lambda_{m}+1\right)}, \\
& 0<x<\infty, \quad \lambda_{m}>0, \quad m=1,2,3,
\end{aligned}
$$

and $q_{v}(\mathbf{\Psi} \mid \mathbf{y})$ is defined as shown in Box III. Thus, the posterior predictive distribution given in Eq. (33), assuming the UP and the JP of a future observation, $X=Y_{n+1}$, for the given data, $\mathbf{y}$, is given by:

$$
\begin{aligned}
& f(x \mid \mathbf{y})=\frac{\Gamma\left(A_{1 v}+1\right) \Gamma\left(A_{2 v}\right) \Gamma\left(A_{3 v}\right)}{(1+x) \Omega_{v}} \sum_{i=0}^{n-r} \sum_{j=0}^{i} \\
& \left(\begin{array}{c}
n-r \\
i
\end{array}\right)\left(\begin{array}{l}
i \\
j
\end{array}\right)\left(B_{1 v}+\ln (1+x)\right)^{-\left(A_{1 v}+1\right)} \\
& B_{2 v}^{-A_{2 v}} B_{3 v}^{-A_{3 v}} B\left(A_{0 v}+1, C_{0 v}\right) B\left(B_{0 v}, A_{0 v}\right. \\
& \left.+C_{0 v}+1\right)+\frac{\Gamma\left(A_{1 v}\right) \Gamma\left(A_{2 v}+1\right) \Gamma\left(A_{3 v}\right)}{(1+x) \Omega_{v}} \\
& \sum_{i=0}^{n-r} \sum_{j=0}^{i}\left(\begin{array}{c}
n-r \\
i
\end{array}\right)\left(\begin{array}{l}
i \\
j
\end{array}\right) B_{1 v}^{-A_{1 v}}\left(B_{2 v}\right. \\
& +\ln (1+x))^{-\left(A_{2 v}+1\right)} B_{3 v}^{-A_{3 v}} B\left(A_{0 v},\right. \\
& \left.C_{0 v}\right) B\left(B_{0 v}+1, A_{0 v}+C_{0 v}+1\right) \\
& +\frac{\Gamma\left(A_{1 v}\right) \Gamma\left(A_{2 v}\right) \Gamma\left(A_{3 v}+1\right)}{(1+x) \Omega_{v}} \sum_{i=0}^{n-r} \sum_{j=0}^{i} \\
& \left(\begin{array}{c}
n-r \\
i
\end{array}\right)\left(\begin{array}{c}
i \\
j
\end{array}\right) B_{1 v}^{-A_{1 v}} B_{2 v}^{-A_{2 v}}\left(B_{3 v}\right. \\
& +\ln (1+x))^{-\left(A_{3 v}+1\right)} B\left(A_{0 v}+1, C_{0 v}\right. \\
& +1) B\left(B_{0 v}, A_{0 v}+C_{0 v}+1\right) \text {. }
\end{aligned}
$$

To construct a Bayesian predictive interval, suppose $L$ and $U$ are the two endpoints of the Bayesian predictive

$$
q_{v}(\mathbf{\Psi} \mid \mathbf{y})=\frac{\sum_{i=0}^{n-r} \sum_{j=0}^{i}\left(\begin{array}{c}
n-r \\
i
\end{array}\right)\left(\begin{array}{c}
i \\
j
\end{array}\right) \exp \left(-B_{1 v} \lambda_{1}\right) \exp \left(-B_{2 v} \lambda_{2}\right) \exp \left(-B_{3 v} \lambda_{3}\right) p_{1}^{A_{0 v}-1} p_{2}^{B_{0 v}-1}\left(1-p_{1}-p_{2}\right)^{C_{0 v}-1}}{\Omega_{v} \lambda_{1}^{1-A_{1 v}} \lambda_{2}^{1-A_{2 v}} \lambda_{3}^{1-A_{3 v}}}
$$


interval. These two endpoints can be obtained using the posterior predictive distribution defined in Eq. (34). A $100(1-\propto) \%$ Bayesian predictive interval $(L, U)$ can be obtained by solving the following equations:

$$
\int_{0}^{L} f(x \mid \mathbf{y}) d x=\frac{\alpha}{2}=\int_{U}^{\infty} f(x \mid \mathbf{y}) d x
$$

or:

$$
\begin{aligned}
& \frac{\Gamma\left(A_{1 v}+1\right) \Gamma\left(A_{2 v}\right) \Gamma\left(A_{3 v}\right)}{A_{1 v} \Omega_{v}} \sum_{i=0}^{n-r} \sum_{j=0}^{i}\left(\begin{array}{c}
n-r \\
i
\end{array}\right)\left(\begin{array}{l}
i \\
j
\end{array}\right) \\
& \left\{B_{1 v}^{-A_{1 v}}-\left(B_{1 v}+\ln (1+L)\right)^{-A_{1 v}}\right\} \\
& B_{2 v}^{-A_{2 v}} B_{3 v}^{-A_{3 v}} B\left(A_{0 v}+1, C_{0 v}\right) B\left(B_{0 v}, A_{0 v}+C_{0 v}\right. \\
& +1)+\frac{\Gamma\left(A_{1 v}\right) \Gamma\left(A_{2 v}+1\right) \Gamma\left(A_{3 v}\right)}{A_{2 v} \Omega_{v}} \sum_{i=0}^{n-r} \sum_{j=0}^{i}\left(\begin{array}{c}
n-r \\
i
\end{array}\right) \\
& \left(\begin{array}{l}
i \\
j
\end{array}\right) B_{1 v}^{-A_{1 v}}\left\{B_{2 v}^{-A_{2 v}}-\left(B_{2 v}+\ln (1+L)\right)^{-A_{2 v}}\right\} \\
& B_{3 v}^{-A_{3 v}} B\left(A_{0 v}, C_{0 v}\right) B\left(B_{0 v}+1, A_{0 v}+C_{0 v}+1\right) \\
& +\frac{\Gamma\left(A_{1 v}\right) \Gamma\left(A_{2 v}\right) \Gamma\left(A_{3 v}+1\right)}{A_{3 v} \Omega_{v}} \sum_{i=0}^{n-r} \sum_{j=0}^{i}\left(\begin{array}{c}
n-r \\
i
\end{array}\right)\left(\begin{array}{l}
i \\
j
\end{array}\right) \\
& B_{1 v}^{-A_{1 v}} B_{2 v}^{-A_{2 v}}\left\{B_{3 v}^{-A_{3 v}}-\left(B_{3 v}+\ln (1+L)\right)^{-A_{3 v}}\right\} \\
& B\left(A_{0 v}, C_{0 v}+1\right) B\left(B_{0 v}, A_{0 v}+C_{0 v}+1\right)=\frac{\alpha}{2}
\end{aligned}
$$

and:

$$
\begin{gathered}
\frac{\Gamma\left(A_{1 v}+1\right) \Gamma\left(A_{2 v}\right) \Gamma\left(A_{3 v}\right)}{A_{1 v} \Omega_{v}} \sum_{i=0}^{n-r} \sum_{j=0}^{i}\left(\begin{array}{c}
n-r \\
i
\end{array}\right)\left(\begin{array}{l}
i \\
j
\end{array}\right) \\
\left(B_{1 v}+\ln (1+U)\right)^{-A_{1 v}} B_{2 v}^{-A_{2 v}} B_{3 v}^{-A_{3 v}} \\
B\left(A_{0 v}+1, C_{0 v}\right) B\left(B_{0 v}, A_{0 v}+C_{0 v}+1\right) \\
+\frac{\Gamma\left(A_{1 v}\right) \Gamma\left(A_{2 v}+1\right) \Gamma\left(A_{3 v}\right)}{A_{2 v} \Omega_{v}} \sum_{i=0}^{n-r} \sum_{j=0}^{i}\left(\begin{array}{c}
n-r \\
i
\end{array}\right) \\
\left(\begin{array}{l}
i \\
j
\end{array}\right) B_{1 v}^{-A_{1 v}}\left(B_{2 v}+\ln (1+U)\right)^{-A_{2 v}} B_{3 v}^{-A_{3 v}} \\
B\left(A_{0 v}, C_{0 v}\right) B\left(B_{0 v}+1, A_{0 v}+C_{0 v}+1\right)
\end{gathered}
$$

$$
\begin{aligned}
& +\frac{\Gamma\left(A_{1 v}\right) \Gamma\left(A_{2 v}\right) \Gamma\left(A_{3 v}+1\right)}{A_{3 v} \Omega_{v}} \sum_{i=0}^{n-r} \sum_{j=0}^{i}\left(\begin{array}{c}
n-r \\
i
\end{array}\right) \\
& \left(\begin{array}{c}
i \\
j
\end{array}\right) B_{1 v}^{-A_{1 v}} B_{2 v}^{-A_{2 v}}\left(B_{3 v}+\ln (1+U)\right)^{-A_{3 v}} \\
& B\left(A_{0 v}, C_{0 v}+1\right) B\left(B_{0 v}, A_{0 v}+C_{0 v}+1\right)=\frac{\alpha}{2} .
\end{aligned}
$$

\begin{tabular}{|c|c|}
\hline \multicolumn{2}{|c|}{ Posterior risks } \\
\hline UP & JP \\
\hline $\lim _{t \rightarrow \infty} \rho\left(\hat{\lambda}_{1}\right)=\frac{n_{1}+1}{\left(\sum_{k=1}^{n_{1}} \ln \left(1+y_{1 k}\right)\right)^{2}}$ & $\lim _{t \rightarrow \infty} \rho\left(\hat{\lambda}_{1}\right)=\frac{n_{1}}{\left(\sum_{k=1}^{n_{1}} \ln \left(1+y_{1 k}\right)\right)^{2}}$ \\
\hline $\lim _{t \rightarrow \infty} \rho\left(\hat{\lambda}_{2}\right)=\frac{n_{2}+1}{\left(\sum_{k=1}^{n_{2}} \ln \left(1+y_{2 k}\right)\right)^{2}}$ & $\lim _{t \rightarrow \infty} \rho\left(\hat{\lambda}_{2}\right)=\frac{n_{2}}{\left(\sum_{k=1}^{n_{2}} \ln \left(1+y_{2 k}\right)\right)^{2}}$ \\
\hline $\lim _{t \rightarrow \infty} \rho\left(\hat{\lambda}_{3}\right)=\frac{n_{3}+1}{\left(\sum_{k=1}^{n_{3}} \ln \left(1+y_{3 k}\right)\right)^{2}}$ & $\lim _{t \rightarrow \infty} \rho\left(\hat{\lambda}_{3}\right)=\frac{n_{3}}{\left(\sum_{k=1}^{n_{3}} \ln \left(1+y_{3 k}\right)\right)^{2}}$ \\
\hline $\lim _{t \rightarrow \infty} \rho\left(\hat{p}_{1}\right)=\frac{\left(n_{1}+1\right)\left(n_{2}+n_{3}+2\right)}{(n+3)^{2}(n+4)}$ & $\lim _{t \rightarrow \infty} \rho\left(\hat{p}_{1}\right)=\frac{\left(n_{1}+1\right)\left(n_{2}+n_{3}+2\right)}{(n+3)^{2}(n+4)}$ \\
\hline $\lim _{t \rightarrow \infty} \rho\left(\hat{p}_{2}\right)=\frac{\left(n_{2}+1\right)\left(n_{1}+n_{3}+2\right)}{(n+3)^{2}(n+4)}$ & $\lim _{t \rightarrow \infty} \rho\left(\hat{p}_{2}\right)=\frac{\left(n_{2}+1\right)\left(n_{1}+n_{3}+2\right)}{(n+3)^{2}(n+4)}$ \\
\hline
\end{tabular}

\section{Limiting expressions for complete dataset}

When test termination time $t$ tends to $\infty$, uncensored observations $r$ tends to sample size $n$ and $r_{l}$ tends to $n_{l}$, $l=1,2,3$. Consequently, all the censored observations become uncensored and the amount of information contained in the sample increases and results in the reduction of the posterior risks of the Bayes estimators. Thus, efficiency of the Bayes estimators increases, because all the observations are incorporated in our sample (see $[4,52])$. When $t$ tends to $\infty$, the limiting expressions for the Bayes estimators and posterior risks are given in Tables 2-7.

Table 2. Limiting expressions for the Bayes estimators assuming the UP and the JP under SELF.

\begin{tabular}{ll}
\hline \multicolumn{3}{c}{ Bayes estimators } \\
\hline $\lim _{t \rightarrow \infty} \hat{\lambda}_{1}=\frac{n_{1}+1}{\sum_{k=1}^{n_{1}} \ln \left(1+y_{1 k}\right)}$ & $\lim _{t \rightarrow \infty} \hat{\lambda}_{1}=\frac{n_{1}}{\sum_{k=1}^{n_{1}} \ln \left(1+y_{1 k}\right)}$ \\
$\lim _{t \rightarrow \infty} \hat{\lambda}_{2}=\frac{n_{2}+1}{\sum_{k=1}^{n_{2}} \ln \left(1+y_{2 k}\right)}$ & $\lim _{t \rightarrow \infty} \hat{\lambda}_{2}=\frac{n_{2}}{\sum_{k=1}^{n_{2}} \ln \left(1+y_{2 k}\right)}$ \\
$\lim _{t \rightarrow \infty} \hat{\lambda}_{3}=\frac{n_{3}+1}{\sum_{k=1}^{n_{3}} \ln \left(1+y_{3 k}\right)}$ & $\lim _{t \rightarrow \infty} \hat{\lambda}_{3}=\frac{n_{3}}{\sum_{k=1}^{n_{3}} \ln \left(1+y_{3 k}\right)}$ \\
$\lim _{t \rightarrow \infty} \hat{p}_{1}=\frac{n_{1}+1}{n+3}$ & $\lim _{t \rightarrow \infty} \hat{p}_{1}=\frac{n_{1}+1}{n+3}$ \\
$\lim _{t \rightarrow \infty} \hat{p}_{2}=\frac{n_{2}+1}{n+3}$ & $\lim _{t \rightarrow \infty} \hat{p}_{2}=\frac{n_{2}+1}{n+3}$ \\
\hline
\end{tabular}

Table 3. Limiting expressions for the posterior risks assuming the UP and the JP under SELF. 
Table 4. Limiting expressions for the Bayes estimators assuming the UP and the JP under PLF.

\begin{tabular}{|c|c|}
\hline \multicolumn{2}{|c|}{ Bayes estimators } \\
\hline UP & JP \\
\hline $\lim _{t \rightarrow \infty} \hat{\lambda}_{1}=\frac{\left(n_{1}+1\right)^{1 / 2}\left(n_{1}+2\right)^{1 / 2}}{\left(\sum_{k=1}^{n_{1}} \ln \left(1+y_{1 k}\right)\right)^{1 / 2}}$ & $\lim _{t \rightarrow \infty} \hat{\lambda}_{1}=\frac{\left(n_{1}\right)^{1 / 2}\left(n_{1}+1\right)^{1 / 2}}{\left(\sum_{k=1}^{n_{1}} \ln \left(1+y_{1 k}\right)\right)^{1 / 2}}$ \\
\hline $\lim _{t \rightarrow \infty} \hat{\lambda}_{2}=\frac{\left(n_{2}+1\right)^{1 / 2}\left(n_{2}+2\right)^{1 / 2}}{\left(\sum_{k=1}^{n_{2}} \ln \left(1+y_{2 k}\right)\right)^{1 / 2}}$ & $\lim _{t \rightarrow \infty} \hat{\lambda}_{2}=\frac{\left(n_{2}\right)^{1 / 2}\left(n_{2}+1\right)^{1 / 2}}{\left(\sum_{k=1}^{n_{2}} \ln \left(1+y_{2 k}\right)\right)^{1 / 2}}$ \\
\hline $\lim _{t \rightarrow \infty} \hat{\lambda}_{3}=\frac{\left(n_{3}+1\right)^{1 / 2}\left(n_{3}+2\right)^{1 / 2}}{\left(\sum_{k=1}^{n_{3}} \ln \left(1+y_{3 k}\right)\right)^{1 / 2}}$ & $\lim _{t \rightarrow \infty} \hat{\lambda}_{3}=\frac{\left(n_{3}\right)^{1 / 2}\left(n_{3}+1\right)^{1 / 2}}{\left(\sum_{k=1}^{n_{3}} \ln \left(1+y_{3 k}\right)\right)^{1 / 2}}$ \\
\hline $\lim _{t \rightarrow \infty} \hat{p}_{1}=\frac{\left(n_{1}+1\right)^{1 / 2}\left(n_{1}+2\right)^{1 / 2}}{(n+3)^{1 / 2}(n+4)^{1 / 2}}$ & $\lim _{t \rightarrow \infty} \hat{p}_{1}=\frac{\left(n_{1}+1\right)^{1 / 2}\left(n_{1}+2\right)^{1 / 2}}{(n+3)^{1 / 2}(n+4)^{1 / 2}}$ \\
\hline $\lim _{t \rightarrow \infty} \hat{p}_{2}=\frac{\left(n_{2}+1\right)^{1 / 2}\left(n_{2}+2\right)^{1 / 2}}{(n+3)^{1 / 2}(n+4)^{1 / 2}}$ & $\lim _{t \rightarrow \infty} \hat{p}_{2}=\frac{\left(n_{2}+1\right)^{1 / 2}\left(n_{2}+2\right)^{1 / 2}}{(n+3)^{1 / 2}(n+4)^{1 / 2}}$ \\
\hline
\end{tabular}

\section{Simulation study}

It is obvious that the analytical comparisons among the Bayes estimators (under different priors and loss functions) are not possible; a simulation study is conducted to serve this purpose. The performance of Bayes estimators has been scrutinized under different priors, loss functions, parametric values, sample sizes, and test termination times. We calculated the Bayes estimates and posterior risks of five parameters $\lambda_{1}, \lambda_{2}, \lambda_{3}, p_{1}$, and $p_{2}$ of a 3-component mixture of Burr distributions given in Eqs. (1) and (4) through a Monte Carlo simulation using the following steps.

1. A random sample of the mixtures is generated as follows:

(i) For each observation, a random number, $u$, is generated from the uniform distribution over the interval $(0,1)$;

(ii) If $u<p_{1}$, then a random variate, $y$, is generated by using Eq. (4) as $y=F_{1}^{-1}(u)$ (the cdf of Burr distribution with parameter $\lambda_{1}$ );

(iii) If $p_{1}<u<p_{2}$, then a random variate, $y$, is generated by using Eq. (4) as $y=F_{2}^{-1}(u)$ (the cdf of Burr distribution with parameter $\left.\lambda_{2}\right)$;
Table 6. Limiting expressions for the Bayes estimators assuming the UP and the JP under DLF.

\begin{tabular}{ll}
\hline \multicolumn{3}{c}{ Bayes estimators } \\
\hline $\lim _{t \rightarrow \infty} \hat{\lambda}_{1}=\frac{n_{1}+2}{\sum_{k=1}^{n_{1}} \ln \left(1+y_{1 k}\right)}$ & $\lim _{t \rightarrow \infty} \hat{\lambda}_{1}=\frac{n_{1}+1}{\sum_{k=1}^{n_{1}} \ln \left(1+y_{1 k}\right)}$ \\
$\lim _{t \rightarrow \infty} \hat{\lambda}_{2}=\frac{n_{2}+2}{\sum_{k=1}^{n_{2}} \ln \left(1+y_{2 k}\right)}$ & $\lim _{t \rightarrow \infty} \hat{\lambda}_{2}=\frac{n_{2}+1}{\sum_{k=1}^{n_{2}} \ln \left(1+y_{2 k}\right)}$ \\
$\lim _{t \rightarrow \infty} \hat{\lambda}_{3}=\frac{n_{3}+2}{\sum_{k=1}^{n_{3}} \ln \left(1+y_{3 k}\right)}$ & $\lim _{t \rightarrow \infty} \hat{\lambda}_{3}=\frac{n_{3}+1}{\sum_{k=1}^{n_{3}} \ln \left(1+y_{3 k}\right)}$ \\
$\lim _{t \rightarrow \infty} \hat{p}_{1}=\frac{n_{1}+2}{n+4}$ & $\lim _{t \rightarrow \infty} \hat{p}_{1}=\frac{n_{1}+2}{n+4}$ \\
$\lim _{t \rightarrow \infty} \hat{p}_{2}=\frac{n_{2}+2}{n+4}$ & $\lim _{t \rightarrow \infty} \hat{p}_{2}=\frac{n_{2}+2}{n+4}$ \\
\hline
\end{tabular}

Table 7. Limiting expressions for the posterior risks assuming the UP and the JP under DLF.

\begin{tabular}{cc}
\hline \multicolumn{2}{c}{ Posterior risks } \\
\hline $\lim _{t \rightarrow \infty} \rho\left(\hat{\lambda}_{1}\right)=\frac{1}{n_{1}+2}$ & JP \\
$\lim _{t \rightarrow \infty} \rho\left(\hat{\lambda}_{2}\right)=\frac{1}{n_{2}+2}$ & $\lim _{t \rightarrow \infty} \rho\left(\hat{\lambda}_{2}\right)=\frac{1}{n_{1}+1}$ \\
$\lim _{t \rightarrow \infty} \rho\left(\hat{\lambda}_{3}\right)=\frac{1}{n_{3}+1}$ & $\lim _{t \rightarrow \infty} \rho\left(\hat{\lambda}_{3}\right)=\frac{1}{n_{3}+1}$ \\
$\lim _{t \rightarrow \infty} \rho\left(\hat{p}_{1}\right)=\frac{\left(n_{2}+n_{3}+2\right)}{\left(n_{1}+2\right)(n+3)}$ & $\lim _{t \rightarrow \infty} \rho\left(\hat{p}_{1}\right)=\frac{\left(n_{2}+n_{3}+2\right)}{\left(n_{1}+2\right)(n+3)}$ \\
$\lim _{t \rightarrow \infty} \rho\left(\hat{p}_{2}\right)=\frac{\left(n_{1}+n_{3}+2\right)}{\left(n_{2}+2\right)(n+3)}$ & $\lim _{t \rightarrow \infty} \rho\left(\hat{p}_{2}\right)=\frac{\left(n_{1}+n_{3}+2\right)}{\left(n_{2}+2\right)(n+3)}$ \\
\hline
\end{tabular}

(iv) If $u>p_{2}$, then a random variate, $y$, is generated by using Eq. (4) as $y=F_{3}^{-1}(u)$ (the cdf of Burr distribution with parameter $\left.\lambda_{3}\right)$.

2. A sample censored at a fixed test termination time, $t$, is selected. The observations which are greater than a fixed test termination time, $t$, are taken as censored ones;

3. Using the steps 1 and 2 for the fixed values of

Table 5. Limiting expressions for the posterior risks assuming the UP and the JP under PLF.

\begin{tabular}{ccc}
\hline UP & Posterior risks \\
\hline $\lim _{t \rightarrow \infty} \rho\left(\hat{\lambda}_{1}\right)=\frac{2\left(n_{1}+1\right)}{\sum_{k=1}^{n_{1}} \ln \left(1+y_{1}\right)}\left\{\frac{\left(n_{1}+2\right)^{1 / 2}}{\left(n_{1}+1\right)^{1 / 2}}-1\right\}$ & $\lim _{t \rightarrow \infty} \rho\left(\hat{\lambda}_{1}\right)=\frac{2 n_{1}}{\sum_{k=1}^{n_{1}} \ln \left(1+y_{1}\right)}\left\{\frac{\left(n_{1}+1\right)^{1 / 2}}{\left(n_{1}\right)^{1 / 2}}-1\right\}$ \\
$\lim _{t \rightarrow \infty} \rho\left(\hat{\lambda}_{2}\right)=\frac{2\left(n_{2}+1\right)}{\sum_{k=1}^{n_{2}} \ln \left(1+y_{2 k}\right)}\left\{\frac{\left(n_{2}+2\right)^{1 / 2}}{\left(n_{2}+1\right)^{1 / 2}}-1\right\}$ & $\lim _{t \rightarrow \infty} \rho\left(\hat{\lambda}_{2}\right)=\frac{2 n_{2}}{\sum_{k=1}^{n_{2}} \ln \left(1+y_{2 k}\right)}\left\{\frac{\left(n_{2}+1\right)^{1 / 2}}{\left(n_{2}\right)^{1 / 2}}-1\right\}$ \\
$\lim _{t \rightarrow \infty} \rho\left(\hat{\lambda}_{3}\right)=\frac{2\left(n_{3}+1\right)}{\sum_{k=1}^{n_{3}} \ln \left(1+y_{3}\right)}\left\{\frac{\left(n_{3}+2\right)^{1 / 2}}{\left(n_{3}+1\right)^{1 / 2}}-1\right\}$ & $\lim _{t \rightarrow \infty} \rho\left(\hat{\lambda}_{3}\right)=\frac{2 n_{3}}{\sum_{k=1}^{n_{3}} \ln \left(1+y_{3 k}\right)}\left\{\frac{\left(n_{3}+1\right)^{1 / 2}}{\left(3_{2}\right)^{1 / 2}}-1\right\}$ \\
$\lim _{t \rightarrow \infty} \rho\left(\hat{p}_{1}\right)=\frac{2\left(n_{1}+1\right)}{(n+3)}\left\{\frac{\left(n_{1}+2\right)^{1 / 2}\left(n_{1}+1\right)^{-1 / 2}}{(n+4)^{1 / 2}(n+3)^{-1 / 2}}-1\right\}$ & $\lim _{t \rightarrow \infty} \rho\left(\hat{p}_{1}\right)=\frac{2\left(n_{1}+1\right)}{(n+3)}\left\{\frac{\left(n_{1}+2\right)^{1 / 2}\left(n_{1}+1\right)^{-1 / 2}}{(n+4)^{1 / 2}(n+3)^{-1 / 2}}-1\right\}$ \\
$\lim _{t \rightarrow \infty} \rho\left(\hat{p}_{2}\right)=\frac{2\left(n_{2}+1\right)}{(n+3)}\left\{\frac{\left(n_{2}+2\right)^{1 / 2}\left(n_{2}+1\right)^{-1 / 2}}{(n+4)^{1 / 2}(n+3)^{-1 / 2}}-1\right\}$ & $\lim _{t \rightarrow \infty} \rho\left(\hat{p}_{2}\right)=\frac{2\left(n_{2}+1\right)}{(n+3)}\left\{\frac{\left(n_{2}+2\right)^{1 / 2}\left(n_{2}+1\right)^{-1 / 2}}{(n+4)^{1 / 2}(n+3)^{-1 / 2}}-1\right\}$ \\
\hline
\end{tabular}


Table 8. Bayes Estimate (BE) and Posterior Risk (PR) using the UP with $\lambda_{1}=6, \lambda_{2}=5, \lambda_{3}=4, p_{1}=0.5$, and $p_{2}=0.3$.

\begin{tabular}{|c|c|c|c|c|c|c|c|c|}
\hline \multirow{2}{*}{$l$} & \multirow{2}{*}{$n$} & \multirow{2}{*}{\multicolumn{2}{|c|}{ Loss functions }} & \multicolumn{5}{|c|}{ UP } \\
\hline & & & & $\hat{\lambda}_{1}$ & $\hat{\lambda}_{2}$ & $\hat{\lambda}_{3}$ & $\hat{p}_{1}$ & $\hat{p}_{2}$ \\
\hline \multirow{18}{*}{0.4} & \multirow{6}{*}{50} & \multirow{2}{*}{ SELF } & $\mathrm{BE}$ & 6.997260 & 6.691200 & 6.452920 & 0.489451 & 0.303943 \\
\hline & & & PR & 4.583960 & 7.799900 & 13.13170 & 0.006500 & 0.005796 \\
\hline & & \multirow{2}{*}{ PLF } & $\mathrm{BE}$ & 7.348310 & 7.177890 & 7.604400 & 0.496734 & 0.314146 \\
\hline & & & $\mathrm{PR}$ & 0.608363 & 1.018170 & 1.685550 & 0.013223 & 0.018654 \\
\hline & & \multirow{2}{*}{ DLF } & $\mathrm{BE}$ & 7.602530 & 7.528110 & 8.423640 & 0.505724 & 0.323751 \\
\hline & & & $\mathrm{PR}$ & 0.081876 & 0.143088 & 0.214871 & 0.026505 & 0.059566 \\
\hline & \multirow{6}{*}{100} & \multirow{2}{*}{ SELF } & $\mathrm{BE}$ & 6.538970 & 5.869170 & 5.618080 & 0.496233 & 0.303230 \\
\hline & & & $\mathrm{PR}$ & 2.186560 & 3.444070 & 5.482010 & 0.003609 & 0.003254 \\
\hline & & \multirow{2}{*}{ PLF } & $\mathrm{BE}$ & 6.777700 & 6.231320 & 5.917420 & 0.498822 & 0.306856 \\
\hline & & & $\mathrm{PR}$ & 0.322962 & 0.555434 & 0.854331 & 0.007218 & 0.010590 \\
\hline & & \multirow{2}{*}{ DLF } & $\mathrm{BE}$ & 6.803540 & 6.629790 & 6.353780 & 0.505517 & 0.309445 \\
\hline & & & PR & 0.048157 & 0.089911 & 0.145986 & 0.014627 & 0.035049 \\
\hline & \multirow{6}{*}{200} & \multirow{2}{*}{ SELF } & $\mathrm{BE}$ & 6.287410 & 5.487840 & 4.825760 & 0.497838 & 0.301859 \\
\hline & & & PR & 1.124150 & 1.727490 & 2.467200 & 0.001986 & 0.001804 \\
\hline & & \multirow{2}{*}{ PLF } & $\mathrm{BE}$ & 6.410030 & 5.681020 & 5.079700 & 0.499043 & 0.304370 \\
\hline & & & PR & 0.174347 & 0.301519 & 0.474581 & 0.003956 & 0.005892 \\
\hline & & \multirow{2}{*}{ DLF } & $\mathrm{BE}$ & 6.505640 & 5.754450 & 5.421110 & 0.502216 & 0.308710 \\
\hline & & & PR & 0.026876 & 0.052632 & 0.091868 & 0.007846 & 0.019183 \\
\hline \multirow{18}{*}{0.7} & \multirow{6}{*}{50} & \multirow{2}{*}{ SELF } & $\mathrm{BE}$ & 6.722710 & 6.012760 & 5.576240 & 0.492014 & 0.302771 \\
\hline & & & PR & 2.550230 & 3.742480 & 5.365850 & 0.004976 & 0.004285 \\
\hline & & \multirow{2}{*}{ PLF } & $\mathrm{BE}$ & 6.813420 & 6.272540 & 6.039290 & 0.497186 & 0.310368 \\
\hline & & & PR & 0.36593 & 0.585562 & 0.874647 & 0.010155 & 0.014127 \\
\hline & & \multirow{2}{*}{ DLF } & $\mathrm{BE}$ & 6.956670 & 6.507270 & 6.640960 & 0.501614 & 0.318774 \\
\hline & & & PR & 0.054030 & 0.092403 & 0.141637 & 0.020482 & 0.044960 \\
\hline & \multirow{6}{*}{100} & \multirow{2}{*}{ SELF } & $\mathrm{BE}$ & 6.324060 & 5.480920 & 4.857800 & 0.496323 & 0.301978 \\
\hline & & & PR & 1.237760 & 1.747910 & 2.352140 & 0.002645 & 0.002288 \\
\hline & & \multirow{2}{*}{ PLF } & $\mathrm{BE}$ & 6.416690 & 5.696410 & 5.019710 & 0.499082 & 0.305470 \\
\hline & & & PR & 0.188655 & 0.303863 & 0.438772 & 0.005299 & 0.007493 \\
\hline & & \multirow{2}{*}{ DLF } & $\mathrm{BE}$ & 6.524860 & 5.873160 & 5.230060 & 0.501384 & 0.308068 \\
\hline & & & PR & 0.029086 & 0.052747 & 0.085033 & 0.010579 & 0.024499 \\
\hline & & SEIF & $\mathrm{BE}$ & 6.152260 & 5.292670 & 4.468900 & 0.498232 & 0.300575 \\
\hline & & & PR & 0.606736 & 0.861029 & 1.061440 & 0.001359 & 0.001177 \\
\hline & 200 & PIF & $\mathrm{BE}$ & 6.245260 & 5.362640 & 4.599630 & 0.499281 & 0.303342 \\
\hline & & & PR & 0.098646 & 0.159784 & 0.231530 & 0.002727 & 0.003912 \\
\hline & & & $\mathrm{BE}$ & 6.288950 & 5.436710 & 4.687870 & 0.500282 & 0.305337 \\
\hline & & 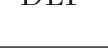 & PR & 0.015778 & 0.029579 & 0.049803 & 0.005462 & 0.012845 \\
\hline
\end{tabular}

parameters, test termination time, and sample size, 1000 samples are generated;

4. The Bayes estimates and posterior risks of parameters $\lambda_{1}, \lambda_{2}, \lambda_{3}, p_{1}$, and $p_{2}$ are calculated based on 1000 Monte Carlo repetitions by solving Eqs. (23)(32).

The above steps 1-4 are used for each of the sample sizes $n=50,100,200$ and each choice of the vector of the parameters $\left(\lambda_{1}, \lambda_{2}, \lambda_{3}, p_{1}, p_{2}\right)=\{(6,5,4,0.5,0.3)$ $(8,7,6,0.5,0.3)\}$ taking $t=0.4,0.7$ The choice of the test termination time is made in such a way that the censoring rate in resulting sample is approximately $10 \%$ to $25 \%$.

From Tables 8-11, it is observed that component parameters $\lambda_{1}, \lambda_{2}, \lambda_{3}$ and the proportion parameter $p_{2}$ are over-estimated assuming the UP and the JP under SELF, PLF, and DLF at different sample sizes and 
Table 9. Bayes Estimate (BE) and Posterior Risk (PR) using the JP with $\lambda_{1}=6, \lambda_{2}=5, \lambda_{3}=4, p_{1}=0.5$, and $p_{2}=0.3$.

\begin{tabular}{|c|c|c|c|c|c|c|c|c|}
\hline \multirow{2}{*}{$l$} & \multirow{2}{*}{$n$} & \multirow{2}{*}{\multicolumn{2}{|c|}{ Loss functions }} & \multicolumn{5}{|c|}{ UP } \\
\hline & & & & $\hat{\lambda}_{1}$ & $\hat{\lambda}_{2}$ & $\hat{\lambda}_{3}$ & $\hat{p}_{1}$ & $\hat{p}_{2}$ \\
\hline \multirow{18}{*}{0.4} & \multirow{6}{*}{50} & \multirow{2}{*}{ SELF } & $\mathrm{BE}$ & 6.886140 & 5.918820 & 5.517360 & 0.484936 & 0.304609 \\
\hline & & & PR & 4.259220 & 6.490770 & 10.56210 & 0.006302 & 0.005679 \\
\hline & & \multirow{2}{*}{ PLF } & $\mathrm{BE}$ & 7.069440 & 6.492790 & 6.093830 & 0.491957 & 0.311387 \\
\hline & & & $\mathrm{PR}$ & 0.570876 & 0.962736 & 1.461740 & 0.012899 & 0.018329 \\
\hline & & \multirow{2}{*}{ DLF } & $\mathrm{BE}$ & 7.476850 & 7.149820 & 6.910650 & 0.497321 & 0.320983 \\
\hline & & & PR & 0.080904 & 0.147252 & 0.237096 & 0.026447 & 0.059106 \\
\hline & \multirow{6}{*}{100} & \multirow{2}{*}{ SELF } & $\mathrm{BE}$ & 6.502440 & 5.630090 & 4.806170 & 0.490801 & 0.302200 \\
\hline & & & PR & 2.134230 & 3.234210 & 4.423630 & 0.003535 & 0.003225 \\
\hline & & \multirow{2}{*}{ PLF } & $\mathrm{BE}$ & 6.737250 & 5.781700 & 5.313280 & 0.494329 & 0.308441 \\
\hline & & & PR & 0.318232 & 0.527867 & 0.827861 & 0.007176 & 0.010588 \\
\hline & & \multirow{2}{*}{ DLF } & $\mathrm{BE}$ & 6.808520 & 6.159180 & 5.810010 & 0.498101 & 0.313483 \\
\hline & & & $\mathrm{PR}$ & 0.047383 & 0.090698 & 0.151750 & 0.014603 & 0.034361 \\
\hline & \multirow{6}{*}{200} & \multirow{2}{*}{ SELF } & $\mathrm{BE}$ & 6.309370 & 5.383100 & 4.516600 & 0.495464 & 0.301536 \\
\hline & & & PR & 1.097750 & 1.642300 & 2.201910 & 0.001937 & 0.001772 \\
\hline & & \multirow{2}{*}{ PLF } & $\mathrm{BE}$ & 6.365650 & 5.534190 & 4.784880 & 0.497933 & 0.303982 \\
\hline & & & PR & 0.170060 & 0.296000 & 0.455499 & 0.003905 & 0.005886 \\
\hline & & \multirow{2}{*}{ DLF } & $\mathrm{BE}$ & 6.511610 & 5.666990 & 4.985940 & 0.499075 & 0.307124 \\
\hline & & & PR & 0.026479 & 0.052774 & 0.093847 & 0.007799 & 0.019238 \\
\hline \multirow{18}{*}{0.7} & \multirow{6}{*}{50} & \multirow{2}{*}{ SELF } & $\mathrm{BE}$ & 6.378500 & 5.639130 & 4.849220 & 0.490331 & 0.302340 \\
\hline & & & PR & 2.365360 & 3.492550 & 4.609880 & 0.004974 & 0.004282 \\
\hline & & \multirow{2}{*}{ PLF } & $\mathrm{BE}$ & 6.495760 & 5.848090 & 5.333080 & 0.495593 & 0.309936 \\
\hline & & & PR & 0.352190 & 0.561249 & 0.830935 & 0.010133 & 0.014048 \\
\hline & & \multirow{2}{*}{ DLF } & $\mathrm{BE}$ & 6.736510 & 6.135530 & 5.741040 & 0.500727 & 0.316377 \\
\hline & & & PR & 0.054126 & 0.096276 & 0.153533 & 0.020386 & 0.045291 \\
\hline & \multirow{6}{*}{100} & \multirow{2}{*}{ SELF } & $\mathrm{BE}$ & 6.207900 & 5.388300 & 4.515750 & 0.495220 & 0.301770 \\
\hline & & & $\mathrm{PR}$ & 1.176680 & 1.675780 & 2.079240 & 0.002623 & 0.002262 \\
\hline & & \multirow{2}{*}{ PLF } & $\mathrm{BE}$ & 6.346240 & 5.520510 & 4.653160 & 0.496980 & 0.305032 \\
\hline & & & PR & 0.186157 & 0.296435 & 0.417369 & 0.005297 & 0.007468 \\
\hline & & \multirow{2}{*}{ DLF } & $\mathrm{BE}$ & 6.372050 & 5.622880 & 5.064280 & 0.500538 & 0.309661 \\
\hline & & & PR & 0.029411 & 0.053728 & 0.089035 & 0.010608 & 0.024308 \\
\hline & & SFIF & $\mathrm{BE}$ & 6.141450 & 5.234200 & 4.264300 & 0.497463 & 0.300380 \\
\hline & & & PR & 0.604560 & 0.848853 & 0.994630 & 0.001353 & 0.001174 \\
\hline & חת & PIF & $\mathrm{BE}$ & 6.171870 & 5.245570 & 4.422600 & 0.499386 & 0.302403 \\
\hline & & & PR & 0.096162 & 0.154823 & 0.222537 & 0.002713 & 0.003891 \\
\hline & & DIF & $\mathrm{BE}$ & 6.222020 & 5.368450 & 4.499850 & 0.500209 & 0.304641 \\
\hline & & & PR & 0.015665 & 0.029549 & 0.050108 & 0.005442 & 0.012821 \\
\hline
\end{tabular}

test termination times. The proportion parameter $p_{1}$ is under-estimated assuming the UP and the JP under SELF and PLF; but, under DLF, it is over-estimated (under-estimated) using the UP (JP) at varying sample sizes and test termination times. The extent of underestimation of component and proportion parameters using the UP and the JP under SELF, PLF, and DLF is lower for larger sample sizes in a fixed test termination time. Also, the extent of over-estimation of component and proportion parameters is higher for smaller test termination times. The extent of over-estimation (under-estimation) of component and proportion parameters is higher for smaller values of component parameters at varying test termination times and sample sizes. The differences of the Bayes estimates of component and proportion parameters 
Table 10. Bayes Estimate (BE) and Posterior Risk (PR) using the UP with $\lambda_{1}=8, \lambda_{2}=7, \lambda_{3}=6, p_{1}=0.5$, and $p_{2}=0.3$.

\begin{tabular}{|c|c|c|c|c|c|c|c|c|}
\hline \multirow{2}{*}{$l$} & \multirow{2}{*}{$n$} & \multirow{2}{*}{\multicolumn{2}{|c|}{ Loss functions }} & \multicolumn{5}{|c|}{ UP } \\
\hline & & & & $\hat{\lambda}_{1}$ & $\hat{\lambda}_{2}$ & $\hat{\lambda}_{3}$ & $\hat{p}_{1}$ & $\hat{p}_{2}$ \\
\hline \multirow{18}{*}{0.4} & \multirow{6}{*}{50} & \multirow{2}{*}{ SELF } & $\mathrm{BE}$ & 9.101440 & 8.492790 & 8.341060 & 0.488651 & 0.303173 \\
\hline & & & PR & 5.452830 & 8.613770 & 13.44860 & 0.005265 & 0.004551 \\
\hline & & \multirow{2}{*}{ PLF } & $\mathrm{BE}$ & 9.387530 & 9.211350 & 9.021220 & 0.494763 & 0.309361 \\
\hline & & & PR & 0.557451 & 0.929613 & 1.361310 & 0.010654 & 0.014711 \\
\hline & & \multirow{2}{*}{ DLF } & $\mathrm{BE}$ & 9.499820 & 9.608110 & 9.681080 & 0.502075 & 0.316453 \\
\hline & & & PR & 0.059466 & 0.100828 & 0.151129 & 0.021450 & 0.047514 \\
\hline & \multirow{6}{*}{100} & \multirow{2}{*}{ SELF } & $\mathrm{BE}$ & 8.541580 & 7.919530 & 7.329280 & 0.494873 & 0.301242 \\
\hline & & & PR & 2.553910 & 4.068990 & 5.802390 & 0.002794 & 0.002410 \\
\hline & & \multirow{2}{*}{ PLF } & $\mathrm{BE}$ & 8.770320 & 7.970950 & 7.656010 & 0.496402 & 0.306334 \\
\hline & & & PR & 0.292496 & 0.479138 & 0.731082 & 0.005641 & 0.007980 \\
\hline & & \multirow{2}{*}{ DLF } & $\mathrm{BE}$ & 8.780040 & 8.437000 & 8.049320 & 0.500752 & 0.309444 \\
\hline & & & $\mathrm{PR}$ & 0.033399 & 0.059586 & 0.095045 & 0.011334 & 0.025918 \\
\hline & \multirow{6}{*}{200} & \multirow{2}{*}{ SELF } & $\mathrm{BE}$ & 8.312190 & 7.393980 & 6.726530 & 0.497286 & 0.301108 \\
\hline & & & PR & 1.284600 & 1.937140 & 2.730210 & 0.001458 & 0.001268 \\
\hline & & \multirow{2}{*}{ PLF } & $\mathrm{BE}$ & 8.419090 & 7.594670 & 6.823740 & 0.498471 & 0.302694 \\
\hline & & & PR & 0.154238 & 0.259959 & 0.389658 & 0.002943 & 0.004202 \\
\hline & & \multirow{2}{*}{ DLF } & $\mathrm{BE}$ & 8.404300 & 7.695930 & 7.070130 & 0.500196 & 0.304976 \\
\hline & & & PR & 0.018188 & 0.034037 & 0.056276 & 0.005889 & 0.013827 \\
\hline \multirow{18}{*}{0.7} & \multirow{6}{*}{50} & \multirow{2}{*}{ SELF } & $\mathrm{BE}$ & 8.829450 & 8.229030 & 7.711900 & 0.490367 & 0.302182 \\
\hline & & & PR & 3.654050 & 5.515350 & 7.741740 & 0.004717 & 0.003997 \\
\hline & & \multirow{2}{*}{ PLF } & $\mathrm{BE}$ & 8.911510 & 8.430550 & 8.160940 & 0.495091 & 0.309217 \\
\hline & & & PR & 0.387904 & 0.608145 & 0.872169 & 0.009580 & 0.013098 \\
\hline & & \multirow{2}{*}{ DLF } & $\mathrm{BE}$ & 9.114080 & 8.784570 & 8.431350 & 0.500294 & 0.315044 \\
\hline & & & PR & 0.043606 & 0.072338 & 0.107143 & 0.019265 & 0.042162 \\
\hline & \multirow{6}{*}{100} & \multirow{2}{*}{ SELF } & $\mathrm{BE}$ & 8.316650 & 7.616550 & 6.771110 & 0.495054 & 0.300922 \\
\hline & & & PR & 1.668830 & 2.460720 & 3.105320 & 0.002461 & 0.002082 \\
\hline & & \multirow{2}{*}{ PLF } & $\mathrm{BE}$ & 8.448940 & 7.723530 & 7.025970 & 0.497581 & 0.304828 \\
\hline & & & PR & 0.195833 & 0.306976 & 0.430101 & 0.004952 & 0.006867 \\
\hline & & \multirow{2}{*}{ DLF } & $\mathrm{BE}$ & 8.590020 & 7.941750 & 7.215240 & 0.500142 & 0.307822 \\
\hline & & & PR & 0.023232 & 0.039958 & 0.061849 & 0.009933 & 0.022480 \\
\hline & & SFLF & $\mathrm{BE}$ & 8.243320 & 7.280110 & 6.379610 & 0.497326 & 0.300765 \\
\hline & & & PR & 0.842157 & 1.176740 & 1.465400 & 0.001257 & 0.001065 \\
\hline & 200 & PIF & $\mathrm{BE}$ & 8.255690 & 7.414080 & 6.545220 & 0.498936 & 0.302285 \\
\hline & & & PR & 0.099966 & 0.158283 & 0.221581 & 0.002522 & 0.003528 \\
\hline & & & $\mathrm{BE}$ & 8.305860 & 7.510540 & 6.733040 & 0.500118 & 0.304104 \\
\hline & & DLГ & PR & 0.012069 & 0.021128 & 0.033458 & 0.005047 & 0.011618 \\
\hline
\end{tabular}

from assumed values reduce to zero with an increase in sample size at different test termination times and same is the case for larger test termination times at varying sample sizes.

It can be seen that the posterior risks of Bayes estimators of parameters assuming the UP and the JP under SELF, PLF, and DLF decrease with an increase in sample size for a fixed test termination time. The same observation is made for large test termination times at different sample sizes. Also, the posterior risks of Bayes estimators of component parameters under SELF are small, but the posterior risks of Bayes estimators of component parameters under DLF and the posterior risks of Bayes estimators of proportion parameters under SELF, PLF, and DLF are larger for smaller values of component parameters at different 
Table 11. Bayes Estimate (BE) and Posterior Risk (PR) using the JP with $\lambda_{1}=8, \lambda_{2}=7, \lambda_{3}=6, p_{1}=0.5$, and $p_{2}=0.3$.

\begin{tabular}{|c|c|c|c|c|c|c|c|c|}
\hline \multirow{2}{*}{$l$} & \multirow{2}{*}{$n$} & \multirow{2}{*}{\multicolumn{2}{|c|}{ Loss functions }} & \multicolumn{5}{|c|}{ UP } \\
\hline & & & & $\hat{\lambda}_{1}$ & $\hat{\lambda}_{2}$ & $\hat{\lambda}_{3}$ & $\hat{p}_{1}$ & $\hat{p}_{2}$ \\
\hline \multirow{18}{*}{0.4} & \multirow{6}{*}{50} & \multirow{2}{*}{ SELF } & $\mathrm{BE}$ & 8.641140 & 7.993400 & 7.302960 & 0.486916 & 0.303231 \\
\hline & & & PR & 4.932750 & 7.831050 & 11.54690 & 0.005218 & 0.004507 \\
\hline & & \multirow{2}{*}{ PLF } & $\mathrm{BE}$ & 9.069250 & 8.355610 & 8.051480 & 0.494535 & 0.309745 \\
\hline & & & PR & 0.538713 & 0.881620 & 1.337550 & 0.010562 & 0.014658 \\
\hline & & \multirow{2}{*}{ DLF } & $\mathrm{BE}$ & 9.389360 & 9.167340 & 8.575960 & 0.498551 & 0.315971 \\
\hline & & & PR & 0.059978 & 0.105442 & 0.164111 & 0.021526 & 0.047375 \\
\hline & \multirow{6}{*}{100} & \multirow{2}{*}{ SELF } & $\mathrm{BE}$ & 8.463760 & 7.524270 & 6.767160 & 0.493152 & 0.301301 \\
\hline & & & PR & 2.515380 & 3.785490 & 5.310540 & 0.002779 & 0.002408 \\
\hline & & \multirow{2}{*}{ PLF } & $\mathrm{BE}$ & 8.537750 & 7.926030 & 7.135560 & 0.496273 & 0.305022 \\
\hline & & & PR & 0.283743 & 0.477354 & 0.705170 & 0.005616 & 0.007888 \\
\hline & & \multirow{2}{*}{ DLF } & $\mathrm{BE}$ & 8.741370 & 8.115590 & 7.531480 & 0.498788 & 0.309560 \\
\hline & & & PR & 0.033168 & 0.060628 & 0.098511 & 0.011309 & 0.025798 \\
\hline & \multirow{6}{*}{200} & \multirow{2}{*}{ SELF } & $\mathrm{BE}$ & 8.310270 & 7.296180 & 6.442230 & 0.495855 & 0.301205 \\
\hline & & & PR & 1.268940 & 1.885900 & 2.553720 & 0.001446 & 0.001259 \\
\hline & & \multirow{2}{*}{ PLF } & $\mathrm{BE}$ & 8.340030 & 7.499810 & 6.596130 & 0.498042 & 0.302429 \\
\hline & & & PR & 0.150276 & 0.255460 & 0.377614 & 0.002915 & 0.004164 \\
\hline & & \multirow{2}{*}{ DLF } & $\mathrm{BE}$ & 8.448250 & 7.586900 & 6.780790 & 0.499004 & 0.304982 \\
\hline & & & PR & 0.017835 & 0.033476 & 0.056154 & 0.005836 & 0.013655 \\
\hline \multirow{18}{*}{0.7} & \multirow{6}{*}{50} & \multirow{2}{*}{ SELF } & $\mathrm{BE}$ & 8.531000 & 7.624840 & 6.894530 & 0.489826 & 0.302358 \\
\hline & & & PR & 3.516130 & 5.075680 & 6.841800 & 0.004713 & 0.003995 \\
\hline & & \multirow{2}{*}{ PLF } & $\mathrm{BE}$ & 8.659990 & 7.913270 & 7.389920 & 0.495120 & 0.309025 \\
\hline & & & PR & 0.387680 & 0.603757 & 0.862288 & 0.009579 & 0.013006 \\
\hline & & \multirow{2}{*}{ DLF } & $\mathrm{BE}$ & 8.957510 & 8.200370 & 7.768060 & 0.499207 & 0.315682 \\
\hline & & & PR & 0.044697 & 0.075846 & 0.115101 & 0.019314 & 0.042009 \\
\hline & \multirow{6}{*}{100} & \multirow{2}{*}{ SELF } & $\mathrm{BE}$ & 8.200430 & 7.296860 & 6.414750 & 0.495139 & 0.300922 \\
\hline & & & PR & 1.655160 & 2.356370 & 2.984730 & 0.002460 & 0.002080 \\
\hline & & \multirow{2}{*}{ PLF } & $\mathrm{BE}$ & 8.333100 & 7.501960 & 6.719570 & 0.497522 & 0.304692 \\
\hline & & & PR & 0.195334 & 0.306449 & 0.430046 & 0.004949 & 0.006866 \\
\hline & & \multirow{2}{*}{ DLF } & $\mathrm{BE}$ & 8.487190 & 7.660860 & 6.887420 & 0.499444 & 0.308210 \\
\hline & & & $\mathrm{PR}$ & 0.023485 & 0.040872 & 0.064067 & 0.009949 & 0.022428 \\
\hline & & SFIF & $\mathrm{BE}$ & 8.077800 & 7.239740 & 6.283810 & 0.497620 & 0.300338 \\
\hline & & & PR & 0.808702 & 1.166490 & 1.434430 & 0.001256 & 0.001062 \\
\hline & 200 & PLF & $\mathrm{BE}$ & 8.165370 & 7.298070 & 6.341300 & 0.498864 & 0.302261 \\
\hline & & & PR & 0.099229 & 0.157256 & 0.218314 & 0.002520 & 0.003525 \\
\hline & & DLF & $\mathrm{BE}$ & 8.207960 & 7.294920 & 6.446890 & 0.499748 & 0.304457 \\
\hline & & & PR & 0.012188 & 0.021579 & 0.034558 & 0.005055 & 0.011619 \\
\hline
\end{tabular}

sample sizes and test termination times. However, the posterior risks of Bayes estimators of component parameters under PLF do not follow a pattern.

As far as the problem of selecting a suitable prior is concerned, it can be seen that the JP emerges as a more efficient prior due to less associated posterior risk than that of the UP under both SELF and PLF; but, we cannot identify which prior is suitable under DLF.
On the other hand, the DLF is observed performing superior to PLF and SELF for estimating the component parameters; whereas, for estimating the proportion parameters, SELF is observed performing better than PLF and DLF. The selection of the best prior and loss function does not depend on test termination time and sample size. However, it is to be noted that selection of the best prior (loss function) for a given loss function 
Table 12. Bayesian predictive interval $(L, U)$ using the UP and the JP with $\lambda_{1}=6, \lambda_{2}=5, \lambda_{3}=4, p_{1}=0.5$, and $p_{2}=0.3$.

\begin{tabular}{ccccccc}
\hline \multirow{2}{*}{$\boldsymbol{l}$} & \multirow{n}{*}{$\boldsymbol{n}$} & \multicolumn{2}{c}{ UP } & & \multicolumn{2}{c}{ JP } \\
\cline { 3 - 4 } \cline { 6 - 6 } & & $\boldsymbol{L}$ & $\boldsymbol{U}$ & & $\boldsymbol{L}$ & $\boldsymbol{U}$ \\
\hline \multirow{2}{*}{0.4} & 50 & 0.008340 & 1.078420 & & 0.008993 & 1.266310 \\
& 100 & 0.008950 & 0.970155 & & 0.009284 & 1.040020 \\
& 200 & 0.009286 & 0.893051 & & 0.009451 & 0.922440 \\
\hline \multirow{4}{*}{0.7} & 50 & 0.008642 & 0.929006 & & 0.009194 & 1.045020 \\
& 100 & 0.009114 & 0.868725 & & 0.009398 & 0.917158 \\
& 200 & 0.009420 & 0.838663 & & 0.009563 & 0.861010 \\
\hline
\end{tabular}

Table 13. Bayesian predictive interval $(L, U)$ using the UP and the JP with $\lambda_{1}=8, \lambda_{2}=7, \lambda_{3}=6, p_{1}=0.5$, and $p_{2}=0.3$.

\begin{tabular}{ccccccc}
\hline \multirow{2}{*}{$\boldsymbol{l}$} & \multirow{n}{*}{$\boldsymbol{n}$} & \multicolumn{2}{c}{ UP } & & \multicolumn{2}{c}{ JP } \\
\cline { 3 - 4 } \cline { 6 - 7 } & & $\boldsymbol{L}$ & $\boldsymbol{U}$ & & $\boldsymbol{L}$ & $\boldsymbol{U}$ \\
\hline \multirow{2}{*}{0.4} & 50 & 0.006151 & 0.611745 & & 0.006568 & 0.683404 \\
& 100 & 0.006570 & 0.572961 & & 0.006786 & 0.601959 \\
& 200 & 0.006816 & 0.550113 & & 0.006925 & 0.563462 \\
\hline \multirow{2}{*}{0.7} & 50 & 0.006337 & 0.565627 & & 0.006722 & 0.620488 \\
& 100 & 0.006675 & 0.545038 & & 0.006874 & 0.569459 \\
& 200 & 0.006867 & 0.533859 & & 0.006969 & 0.545406 \\
\hline
\end{tabular}

(prior) is made based on posterior risks associated with it.

The results in Tables 12 and 13 are the $90 \%$ Bayesian predictive intervals assuming the UP and the JP. It is observed that the Bayesian predictive intervals become narrower with an increase in sample size for a fixed test termination time. The same observation can be made with larger test termination times at a fixed sample size. The Bayesian predictive intervals become narrower (wider) for larger (smaller) component parametric values in each sample size and test termination time considered in the simulation study. Also, the Bayesian predictive intervals using the JP are wider than the predictive intervals using the UP.

\section{A real-life example}

Davis [65] reported a mixture data, $\mathbf{x}=\left(x_{11}, x_{12}\right.$, $\left.\ldots, x_{1 r_{1}}, x_{21}, x_{22}, \ldots, x_{2 r_{2}}, x_{31}, x_{32}, \ldots, x_{3 r_{3}}\right)$, on lifetimes (in thousand hours) of many components used in aircraft sets. A part of these data have also been used in [4]. To illustrate the proposed methodology, we take the data on three components, namely, R105 RESISTOR USED IN PE218 CONVERTER, Z303 NETWORK USED IN RF UNIT, and V7 TRANSMITTER TUBE. Davis showed that the data $\mathbf{x}$ could be modeled by a mixture of exponential distributions. The transformation $y=\exp (x)-1$ of an exponential random data $(\mathbf{x})$ yields the Burr random data $(\mathbf{y})$. This transformation allows us to use the Davis mixture data for applying the proposed Bayesian analysis. It is unknown that which component fails until a failure occurs at or before the test termination time (1 hour). The tests are conducted 582 times. The data summary required to evaluate the Bayes estimates and posterior risks is given by:

$$
\begin{aligned}
& n=582, \quad r_{1}=252, \quad r_{2}=54, \quad r_{3}=175 \\
& r=r_{1}+r_{2}+r_{3}=481, \quad n-r=101, \\
& \sum_{k=1}^{r_{1}} \ln \left(1+y_{1 k}\right)=\sum_{k=1}^{r_{1}} x_{1 k}=90.60, \\
& \sum_{k=1}^{r_{2}} \ln \left(1+y_{2 k}\right)=\sum_{k=1}^{r_{2}} x_{2 k}=23.20, \\
& \sum_{k=1}^{r_{3}} \ln \left(1+y_{3 k}\right)=\sum_{k=1}^{r_{3}} x_{3 k}=46.125 .
\end{aligned}
$$

Since $n-r=101$, we have almost $17.35 \%$ type-I right censored sample. The Bayes estimates and their posterior risks are shown in Table 14.

From Table 14, it is noticed that the results obtained through real-life data are compatible with the simulated results. The performance of the Bayes estimators using the JP is seen as the best in comparison with the UP under all the loss functions considered in this study. It is also observed that DLF (SELF) is better than PLF and SELF (PLF and DLF) for estimating component (proportion) parameters.

\section{Conclusion}

A 3-component mixture of Burr distributions is developed to model lifetime data. Type-I right censoring sampling scheme is considered. Assuming the availability of the non-informative priors and different loss functions, expressions of the Bayes estimators and their posterior risks are derived. To judge the relative performance of the Bayes estimators and also to deal with the problem of selecting the priors and loss functions at different sample sizes and test termination times, a comprehensive simulation and reallife study have been conducted. The simulation study revealed some important and interesting properties of the Bayes estimators. From numerical results, we observed that an increase in sample size or test termination time provided improved Bayes estimators. The effect of test termination time, sample size, and parametric values on the Bayes estimators is in the form of over-estimation or under-estimation. To be more specific, the smaller (larger) sample size results 
Table 14. Bayes Estimates (BEs) and Posterior Risks (PRs) assuming the UP and the JP under SELF and DLF with Davis real-life mixture data.

\begin{tabular}{|c|c|c|c|c|c|c|c|}
\hline Prior & Loss & functions & $\hat{\lambda}_{1}$ & $\hat{\lambda}_{2}$ & $\hat{\lambda}_{3}$ & $\hat{p}_{1}$ & $\hat{p}_{2}$ \\
\hline \multirow{4}{*}{ SELF } & \multirow{2}{*}{ UP } & $\mathrm{BE}$ & 1.75303781 & 0.93662706 & 3.32539143 & 0.52658110 & 0.16052665 \\
\hline & & PR & 0.04102084 & 0.07309535 & 0.11130084 & 0.00098376 & 0.00080169 \\
\hline & \multirow{2}{*}{$\mathrm{JP}$} & $\mathrm{BE}$ & 1.76753121 & 0.89014676 & 3.30209669 & 0.52349272 & 0.16349037 \\
\hline & & PR & 0.04066844 & 0.06212724 & 0.11052237 & 0.00095621 & 0.00077923 \\
\hline \multirow{4}{*}{ PLF } & \multirow{2}{*}{ UP } & $\mathrm{BE}$ & 1.76469896 & 0.97486696 & 3.34208453 & 0.52751438 & 0.16300459 \\
\hline & & PR & 0.02332229 & 0.07647981 & 0.03338620 & 0.00186654 & 0.00495588 \\
\hline & \multirow{2}{*}{ JP } & $\mathrm{BE}$ & 1.77899832 & 0.92438546 & 3.31878968 & 0.52440522 & 0.16585636 \\
\hline & & PR & 0.02293422 & 0.06847741 & 0.03338597 & 0.00182501 & 0.00473198 \\
\hline \multirow{4}{*}{ DLF } & \multirow{2}{*}{ UP } & $\mathrm{BE}$ & 1.77643767 & 1.01466809 & 3.35886142 & 0.52844930 & 0.16552078 \\
\hline & & $\mathrm{PR}$ & 0.01317235 & 0.07691287 & 0.00996468 & 0.00353524 & 0.03017225 \\
\hline & \multirow{2}{*}{$\mathrm{JP}$} & $\mathrm{BE}$ & 1.79053982 & 0.95994113 & 3.33556705 & 0.52531932 & 0.16825660 \\
\hline & & $\mathrm{PR}$ & 0.01285010 & 0.07270693 & 0.01003438 & 0.00347712 & 0.02832712 \\
\hline
\end{tabular}

in larger (smaller) extent of over-estimation or underestimation at a fixed test termination time. On the other hand, the extent of over-estimation or underestimation of parameters is quite smaller (larger) with relatively larger (smaller) test termination times for a fixed sample size. Also, the extent of over-estimation or under-estimation of parameters is less for larger values of component parameters and vice versa. However, as sample size (test termination time) increases (decreases), the posterior risks of Bayes estimators of parameters decrease (increase) for a fixed test termination time (sample size). As the cut-off test termination time tends to infinity, the limiting expressions (for complete dataset) of the Bayes estimators and posterior risks are greatly simplified. Moreover, the posterior risks of the Bayes estimators (for complete dataset) are expected to reduce further as there is no more effect of test termination time. Finally, we conclude that for a Bayesian analysis of mixture data, the JP paired with SELF and both the UP and the JP paired with DLF are preferable choices for estimating proportion and component parameters, respectively. When PLF is considered, the JP is the suitable prior for estimating component parameters. Also, the results obtained through real-life data coincide with the simulated results.

\section{References}

1. Saleem, M. "Bayesian analysis of mixture distributions", Ph.D. Thesis, Department of Statistics, Quaidi-Azam University, Islamabad, Pakistan (2010).

2. Li, L.A. "Decomposition theorems, conditional probability, and finite mixtures distributions", Thesis, State University, New York, Albany (1983).

3. Li, L.A. and Sedransk, N. "Mixtures of distributions:
A topological approach", The Annals of Statistics, 16(4), pp. 1623-1634 (1988).

4. Tahir, M. and Aslam, M. "On the Bayesian analysis of 3-componen mixture of exponential distribution under different loss functions", Hacettepe Journal of Mathematics and Statistics (2015). DOI: 10.15672/HJMS.2015519451

5. Mendenhall, W. and Hader, R.J. "Estimation of parameters of mixed exponentially distributed failure time distributions from censored life test data", Biometrika, 45(3-4), pp. 504-520 (1958).

6. Rider, P.R. "The method of moments applied to a mixture of two exponential distributions", Annals of Mathematical Statistics, 32, pp. 143-147 (1961).

7. Everitt, B.S. and Hand, D.J., Finite Mixture Distribution, Chapman \& Hall, London (1981).

8. Harris, C.M. "On finite mixtures of geometric and negative binomial distributions", Communications in Statistics-Theory and Methods, 12, pp. 987-1007 (1983).

9. Titterington, D.M., Simth, A.F.M. and Makov, U.E., Statistical Analysis of Finite Mixture Distribution, Wiley, Chichester (1985).

10. Kanji, K.G. "A mixture model for wind shear data", Journal of Applied Statistics, 12, pp. 49-58 (1985).

11. Maclachlan, G.J. and Basford, K.E. "Mixture models: Applications to clustering", Marcel Dekker, New York (1988).

12. Jones, P.N. and McLachlan, J.G. "Laplace-normal mixtures fitted to wind shear data", Journal of Applied Statistics, 17, pp. 271-276 (1990).

13. Lindsay, B.G., Mixture Models: Theory, Geometry and Applications, The Institute of Mathematical Statistics, Hayward, CA (1995).

14. Maclachlan, G.J. and Krishnan, T., The EM Algorithm and Extensions, Wiley, New York (1997). 
15. Maclachlan, G. and Peel, D., Finite Mixture Models, Wiley, New York (2000).

16. AL-Hussaini, E.K. and Sultan, K.S."Reliability and hazard based on finite mixture models", In: Balakrishnan, N. and Rao, C.R. (Eds.), Handbook of Statistics, 20, Elsevier, Amsterdam, pp. 139-183 (2001).

17. Sultan, K.S., Ismail, M.A. and Al-Moisheer, A.S. "Mixture of two inverse Weibull distributions: properties and estimation", Computational Statistics \& Data Analysis, 51(1), pp. 5377-5387 (2007).

18. Abu-Zinadah, H.H. "A study on mixture of exponentiated Pareto and exponential distributions", Journal of Applied Sciences Research, 6(4), pp. 358-376 (2010).

19. Afify, W.M. "Classical estimation of mixed Rayleigh distribution in type-I progressive censored", Journal of Statistical Theory and Applications, 10(4), pp. 619632 (2011).

20. Amin, E.A. "Maximum likelihood estimation of the mixed generalized Rayleigh distribution from typeI censored samples", Applied Mathematical Sciences, 5(56), pp. 2753-2764 (2011).

21. Erişoğlu, Ü., Erişoğlu, M. and Erol, H. "A mixture model of two different distributions approach to the analysis of heterogeneous survival data", World Academy of Science, Engineering and Technology, 54, pp. 41-45 (2011).

22. Kamaruzzaman, Z.A., Isa, Z. and Ismail, M.T. "Mixtures of normal distributions: application to Bursa Malaysia stock market indices", World Applied Sciences Journal, 16(6), pp. 781-790 (2012).

23. McCullagh, P. "Exponential mixtures and quadratic exponential families", Biometrika, 81(4), pp. 721-729 (1994).

24. Sinha, S.K. "Bayesian estimation", New age international (P) limited, publishers, New Delhi (1998).

25. Hebert, J.L. and Scariano, S.M. "Comparing location estimators for exponential mixtures under Pitman's measure of closeness", Communications in StatisticsTheory and Methods, 33(1), pp. 29-46 (2005).

26. Liu, Z. "Bayesian mixture models", MS Thesis, McMaster University, Hamilton, Ontario (2010).

27. Saleem, M. and Irfan, M. "On properties of the Bayes estimates of the Rayleigh mixture parameters: a simulation study", Pakistan Journal of Statistics, 26(3), pp. 547-555 (2010).

28. Saleem, M., Aslam, M. and Economus, P. "On the Bayesian analysis of the mixture of Power distribution using the complete and censored sample", Journal of Applied Statistics, 37(1), pp. 25-40 (2010).

29. Santos, A.M. "Robust estimation of censored mixture models", PhD Thesis, University of Colorado Denver (2011).

30. AL-Hussaini, E.K. and Hussein, M. "Estimation under a finite mixture of exponentiated exponential compo- nents model and balanced square error loss", Open Journal of Statistics, 2, pp. 28-38 (2012),

31. Mohammadi, A. and Salehi-Rad, M.R. "Bayesian inference and prediction in an M/G/1 with optional second service", Communication in Statistics-Simulation and Computation, 41(3), pp. 419-435 (2012).

32. Kazmi, S.M.A., Aslam, M. and Ali, S. "On the Bayesian estimation for two-component mixture of Maxwell distribution, assuming type-I censored data", International Journal of Applied Science and Technology, 2(1), pp. 197-218 (2012).

33. Ahmad, A.E.A. and AL-Zaydi, A.M. "Inferences under a class of finite mixture distributions based on generalized order statistics", Open Journal of Statistics, 3, pp. 231-244 (2013).

34. Ali, S., Aslam, M. and Kazmi, S.M.A. "Choice of suitable informative prior for the scale parameter of the mixture of Laplace distribution", Electronic Journal of Applied Statistical Analysis, 6(1), pp. 32-56 (2013).

35. Mohammadi, A., Salehi-Rad, M.R. and Wit, E.C. "Using mixture of gamma distributions for Bayesian analysis in an M/G/1 queue with optional second service", Computational Statistics, 28(2), pp. 683-700 (2013).

36. Ali, S. "Mixture of the inverse Rayleigh distribution: properties and estimation in Bayesian framework", Applied Mathematical Modelling, 39(2), pp. 515-530 (2014).

37. Ateya, S.F. "Maximum likelihood estimation under a finite mixture of generalized exponential distributions based on censored data", Statistical Papers, 55(2), pp. 311-325 (2014).

38. Feroze, N. and Aslam, M. "Bayesian analysis of doubly censored lifetime data using two-component mixture of Weibull distribution", Journal of the National Science Foundation of Sri Lanka, 42(4), pp. 325-334 (2014).

39. Mohamed, M.M., Saleh, E. and Helmy, S.M. "Bayesian prediction under a finite mixture of generalized exponential lifetime model", Pakistan Journal of Statistics and Operation Research, 10(4), pp. 417-433 (2014).

40. Zhang, H. and Huang, Y. "Finite mixture models and their applications: a review", Austin Biometrics and Biostatistics, 2(1), pp. 1-6 (2015)

41. Romeu, L.J. "Censored data", Strategic Arms Reduction Treaty, 11(3), pp. 1-8 (2004).

42. Gijbels, I. "Censored data", Wiley Interdisciplinary Reviews: Computational Statistics, 2(2), pp. 178-188 (2010).

43. Kalbfleisch, J.D. and Prentice, R.L., The Statistical Analysis of Failure Time Data, 360, John Wiley \& Sons, New York (2011).

44. Burr, I.W. "Cummulative frequency functions", $A n$ nals of Mathematical Statistics, 13, pp. 215-232 (1942).

45. Johnson, N., Kotz, S. and Balakrishnan, N., Continuous Univariate Distributions, John Wiley \& Sons, New York (1994). 
46. Burr, I.W. "On a general system of distributions III, the sample range", Journal of the American Statistical Association, 63, pp. 636-643 (1968).

47. Burr, I.W. "Parameters for a general system of distributions to match a grid of c3 and a4", Communications in Statistics, 2, pp. 1-21 (1973).

48. Burr, I.W. and Cislak, P.J. "On a general system of distributions I. Its curve-shape characteristics. II. The sample median", Journal of the American Statistical Association, 63, pp. 627-635 (1968).

49. Rodriguez, R.N. "A guide to the Burr type-XII distributions", Biometrika, 64, pp. 129-134 (1977).

50. Tadikamalla, P.R. "A look at the Burr and related distributions", International Statistical Review, 48, pp. 337-344 (1980).

51. Economou, P. and Caroni, C. "Graphical tests for the assumption of gamma and inverse Gaussian frailty distributions", Lifetime Data Analysis, 11(4), pp. 565$582(2005)$.

52. Tahir, M. and Aslam, M. "Bayesian analysis of the 3component mixture of exponential distribution assuming the non-informative priors", Revista Colombiana de Estadística, 38(2), pp. 431-452 (2015).

53. Bayes, T. "An essay toward solving a problem in the doctrine of chances", Philosophical Transactions of the Royal Society of London, 53, pp. 370-418 (1763).

54. Geisser, S. "On prior distributions for binary trials", The American Statistician, 38(4), pp. 244-247 (1984).

55. Jeffreys, H. "An invariant form for the prior probability in estimation problems", Proceeding of the Royal Society of London, Mathematical and Physical Sciences, 186(1007), pp. 453-461 (1946).

56. Jeffreys, H., Theory of Probability, Oxford, Claredon Press, UK (1961).

57. Legendre, A.M. "Nouvelles méthodes pour la détermination des orbites des com'etes: appendice sur la meéthode des moindres carŕes", Courcier, Paris (1806).

58. Norstrom, J.G. "The use of precautionary loss function in risk analysis", IEEE Trans. Reliability, 45, pp. 400403 (1996).

59. DeGroot, M.H. Optimal Statistical Decision, John Wiley \& Sons, New York (2005).
60. Arnold, B.C. and Press, S.J. "Bayesian inference for Pareto populations", Journal of Econometrics, 21, pp. 287-306 (1983).

61. Al-Hussaini, E.K., Jaheen, Z.F. and Nigm, A.M. "Bayesian prediction based on finite mixture of Lomax components model and type-I censoring", Statistics, 35, pp. 259-268 (2001).

62. Al-Hussaini, E.K. and Ahmad, A.E.A. "On Bayesian interval prediction of future records", Test, 12, pp. 7999 (2003).

63. Bolstad, W.M. Introduction to Bayesian Statistics, John Wiley \& Sons, New Jersey (2004).

64. Bansal, A.K. Bayesian Parametric Inference, Narosa Publishing House Pvt. Ltd., New Delhi (2007).

65. Davis, D.J. "An analysis of some failure data", Journal of the American Statistical Association, 47(258), pp. 113-150 (1952).

\section{Biographies}

Muhammad Tahir received his MPhil degree in Statistics from the Quaid-i-Azam University, Islamabad, Pakistan. He has published more than 10 research papers in international research journals. Currently, he is a Faculty Member at Government College University, Faisalabad, Pakistan. His research interests include Bayesian inference and mixture distributions.

Muhammad Aslam is a Professor of Statistics at Riphah International University, Islamabad, Pakistan. He has received the $\mathrm{PhD}$ degree in Statistics form University of Wales. He has published over 100 refereed publications and has guided research students at all levels of the curriculum, including undergraduate and $\mathrm{PhD}$. His research interests include Bayesian inference, reliability analysis, and mixture distributions.

Zawar Hussain graduated from Quaid-i-Azam University, Islamabad, Pakistan. He has published 60 research papers in reputed journals. His research interests include sampling techniques, randomized response models, and quality control. 